\title{
Evolutionary synthesis of galaxies at high spectral resolution with the code PEGASE-HR
}

\section{Metallicity and age tracers}

\author{
D. Le Borgne ${ }^{1,2}$, B. Rocca-Volmerange ${ }^{1,7}$, P. Prugniel ${ }^{3}$, A. Lançon ${ }^{4}$, M. Fioc ${ }^{1,6}$, and C. Soubiran ${ }^{5}$ \\ 1 Institut d'Astrophysique de Paris, 98bis boulevard Arago, 75014 Paris, France \\ e-mail: leborgne@iap.fr \\ 2 Dept. of Astronomy \& Astrophysics, University of Toronto, 60 St. George Street, Toronto, ON M5S 3H8, Canada \\ 3 CRAL-Observatoire de Lyon, 9 Av. C. André, 69561 Saint-Genis Laval, France \\ ${ }_{4}$ Observatoire de Strasbourg, 11 rue de l'Université, 67000 Strasbourg, France \\ 5 Observatoire de Bordeaux, BP 89, 33270 Floirac, France \\ ${ }^{6}$ Université Pierre et Marie Curie, 4 place Jussieu, 75005 Paris, France \\ 7 Université Paris-Sud, 91405 Orsay Cedex, France
}

Received 2 May 2003 / Accepted 23 June 2004

\begin{abstract}
We present PEGASE-HR, a new stellar population synthesis program generating high resolution spectra $(R=10000)$ over the optical range $\lambda \lambda=400-680 \mathrm{~nm}$. It links the spectro-photometric model of galaxy evolution PEGASE.2 (Fioc \& Rocca-Volmerange 1997) to an updated version of the ELODIE library of stellar spectra observed with the $193 \mathrm{~cm}$ telescope at the Observatoire de Haute-Provence (Prugniel \& Soubiran 2001a). The ELODIE star set gives a fairly complete coverage of the Hertzprung-Russell (HR) diagram and makes it possible to synthesize populations in the range $[\mathrm{Fe} / \mathrm{H}]=-2$ to +0.4 . This code is an exceptional tool for exploring signatures of metallicity, age, and kinematics. We focus on a detailed study of the sensitivity to age and metallicity of the high-resolution stellar absorption lines and of the classical metallic indices proposed until now to solve the age-metallicity degeneracy. Validity tests on several stellar lines are performed by comparing our predictions for Lick indices to the models of other groups. The comparison with the lower resolution library BaSeL (Lejeune et al. 1997) confirms the quality of the ELODIE library when used for single stellar populations (SSPs) from $10^{7}$ to $2 \times 10^{10}$ yr. Predictions for the evolved populations of globular clusters and elliptical galaxies are given and compared to observational data. Two new highresolution indices are proposed around the $\mathrm{H} \gamma$ line. They should prove useful in the analysis of spectra from the new generation of telescopes and spectrographs.
\end{abstract}

Key words. galaxies: stellar content - galaxies: evolution - galaxies: abundances - techniques: spectroscopic

\section{Introduction}

The rapidly increasing number of high-quality spectroscopic galaxy surveys makes it necessary to improve the galaxy evolution models used for their interpretation. To derive the history of the star formation and the chemical evolution of a stellar population from its line-of-sight integrated spectrum one can either fit spectrophotometric indices and colors with a model, or fit directly the observed spectrum with a synthetic spectral energy distribution (SED).

Spectrophotometric indices characterize the strengths of spectral features that are sensitive to the age or to the metallicity of a stellar population, and generally to both. The indices of Rose et al. (1994) and the Lick indices (Worthey 1994; Kuntschner \& Davies 1998; Trager et al. 2000) are the most widely used. Various models allow us to predict the evolution of these indices. They are based on assumptions for the stellar evolution (evolutionary tracks) and for the history of stellar formation (initial mass function, star formation rate...). They usually require preliminary measurements of the indices in a library of stars. The most recent models (e.g. Thomas et al. 2003) take into account the non-solar abundances resulting from the different processes of metal enrichment by using the response functions of Tripicco \& Bell (1995). Other families of models compute the SED, thus allowing the user to measure indices a posteriori if the spectral resolution is sufficient (e.g., Fioc \& Rocca-Volmerange 1997; Leitherer et al. 1999; Eisenstein et al. 2003; Bruzual \& Charlot 2003). The PEGASE code is one of the latter, and its latest version PEGASE.2 (Fioc \& RoccaVolmerange 1999) is the basis for the present study.

One of the major issues in studying stellar populations is the age-metallicity degeneracy (Worthey 1994). Most indices 
are sensitive to both metallicity and age: a younger age may be confused with a lower metallicity. The comparison of indices featuring Balmer lines with indices of metallic lines may in principle break the degeneracy. But in practice the tests are difficult and give ambiguous results, partly because of the contamination by nebular emission, in particular for $\mathrm{H} \alpha$ and $\mathrm{H} \beta$ (González 1993; Worthey \& Ottaviani 1997; Kuntschner et al. 2001).

The degeneracy can also be partly lifted either by using broad-band colors and indices in an extended range of wavelengths (Gorgas et al. 1993; Worthey et al. 1994) or, more efficiently, by defining indices at a higher spectral resolution (Jones \& Worthey 1995). PEGASE.2, which is based on the library of Lejeune et al. (1997, 1998), is well suited to the first approach. Until recently, such SED-predicting algorithms were unable to reach high spectral resolutions because they lacked adequate stellar spectral libraries.

In a pioneering work, Vazdekis \& Arimoto (1999) demonstrated the potentiality of using SEDs at higher resolution. They used the library of Jones (1998), thus reaching $R=2000-3000$ (the Lick resolution is about 500), and were able to define narrower age-sensitive indices around $\mathrm{H} \gamma$ (with band-widths of 10-20 ̊).

At high resolution, the analysis of a galaxy spectrum must take into account another phenomenon: the internal kinematics broadens the lines and reduces the apparent resolution of the spectrum. Traditionally, measurements of spectrophotometric indices are corrected for velocity dispersion (see e.g. Golev et al. 1999), but for giant elliptical galaxies with typical velocity dispersions $\sigma$ close to $300 \mathrm{~km} \mathrm{~s}^{-1}$, the correction would introduce unacceptable errors for narrow indices. For dwarf galaxies $\left(\sigma<60 \mathrm{~km} \mathrm{~s}^{-1}\right)$ and globular clusters $\left(\sigma<10 \mathrm{~km} \mathrm{~s}^{-1}\right)$, corrections would remain acceptable for indices defined on $2 \AA$-wide passbands.

Synthetic high resolution SEDs are potentially very useful for probing the internal kinematics. They may indeed replace the traditional stellar templates and provide both a measurement of the line-of-sight velocity distribution (LOSVD) and a constraint on the stellar population. Recently, the Vazdekis \& Arimoto (1999) model has been coupled to the Ca II library of Cenarro et al. (2001) to determine the LOSVD profiles of bulges of spiral galaxies (Falcón-Barroso et al. 2003). The LOSVDs are determined by deconvolving the observed spectrum with a template spectrum resulting from the population synthesis program. Using synthesized spectra to study the internal kinematics is not straightforward. Indeed, it is first necessary to convolve the template with the spectral instrumental response, which is generally neither Gaussian nor constant over the whole wavelength range. To do this, the template spectrum must have a significantly higher resolution than the observed one.

To address the questions above, we have coupled the last version of PEGASE to a library of high resolution $(R=10000)$ stellar spectra. In this paper we present the resulting code, PEGASE-HR, and focus on its predictions of line indices that are sensitive to age and metallicity.

In Sect. 2, we recall the main characteristics of PEGASE.2. We also briefly describe the main features of the updated stellar library ELODIE (Prugniel \& Soubiran 2001a) as well as the "interpolator", the method used to convert the set of ELODIE stellar spectra to a grid of spectra with the regularly spaced stellar parameters of the BaSeL library. The synthetic high-resolution spectra of SSPs are then compared to the corresponding low-resolution spectra of PEGASE.2. The variations of the high resolution spectra around commonly exploited lines are illustrated, as a function of both age and metallicity. In Sect. 3, the integrated fluxes, colors and line indices predicted with PEGASE-HR are validated by comparison with previous works. In Sect. 4, as a further test of the model predictions, we compare them to observations of globular clusters and elliptical galaxies. A systematic search for new narrow indices is described in Sect. 5. Perspectives for the new generation of instruments are suggested in the conclusion.

\section{PEGASE-HR: The coupling of PEGASE.2 and ELODIE}

\subsection{PEGASE.2, a spectral evolution code}

The PEGASE. 2 code $^{1}$ is aimed at modeling the spectral evolution of galaxies. It is based on stellar evolutionary tracks from the "Padova" group, extended to the thermally pulsating asymptotic giant branch (AGB) and post-AGB phases; these tracks cover all the masses, metallicities and phases of interest for galaxy spectral synthesis. For a given evolutionary scenario (typically characterized by a star formation law, an initial mass function and, possibly, infall or galactic winds), the code consistently computes the star formation rate and the metallicity of gas and stars at any time. The nebular component (continuum and lines) due to $\mathrm{H}$ in regions is roughly calculated and added to the stellar component. Depending on the type of galaxy (disk or spheroidal), the attenuation of the spectrum by dust is then computed using the output of a radiative transfer code; this code takes into account scattering (Fioc 1997).

PEGASE. 2 uses the BaSeL (Lejeune et al. 1997, 1998) library of stellar spectra and can therefore synthesize lowresolution $(R \simeq 200)$ ultraviolet to near-infrared spectra of Hubble sequence galaxies as well as of starbursts. In PEGASE-HR, the BaSeL library is replaced by a grid of spectra interpolated from the high-resolution ELODIE library of stellar spectra.

\subsection{ELODIE, a high resolution library of stellar spectra}

The ELODIE library is a stellar database of 1959 spectra for 1503 stars, observed with the échelle spectrograph ELODIE on the $193 \mathrm{~cm}$ telescope at the Observatoire de Haute Provence. Previous versions of the library were presented in Soubiran et al. (1998) and Prugniel \& Soubiran (2001a,b). It has been updated for the present work by doubling the number of spectra, which greatly improved the coverage of the parameter space (in effective temperature, surface gravity, and metallicity).

\footnotetext{
1 Version 2 of PEGASE, Projet d'Étude des Galaxies par Synthèse Évolutive in French. The code is available at http://www.iap.fr/pegase/
} 
The data reduction has also been improved, in particular the flux calibration, and the wavelength range has been extended to $\lambda \lambda=400-680 \mathrm{~nm}$. For the purpose of population synthesis, the original resolution $(R=42000)$ has been reduced to $R=10000$ at $\lambda=550 \mathrm{~nm}$, or more precisely to a gaussian instrumental profile of $F W H M \simeq 0.55 \AA$ over the whole range of wavelengths. The typical signal-to-noise $(S / N)$ ratio of the spectra is $500 / \AA$. The connection of several échelle spectra, each one defined in a small wavelength range, is needed to build a single spectrum over the entire range $\lambda \lambda=400-680 \mathrm{~nm}$. The complex procedure used to perform this task leads to an overall (broad-band) photometric precision of $2 \%$. The precision reaches $0.5 \%$ when the ELODIE spectra are normalized to a low resolution continuum, as described in detail in Prugniel \& Soubiran (2001a).

The atmospheric parameters of the stars $\left(T_{\mathrm{eff}}, g,[\mathrm{Fe} / \mathrm{H}]\right)$ are taken from the literature in an up-to-date version of Cayrel de Strobel et al. (2001), when available. Quality weights are applied to the parameters when several estimations were available (for details, see Prugniel \& Soubiran 2001a) ${ }^{2}$. Otherwise they are estimated with the TGMET procedure, which consists in least square fits of the target spectrum to reference spectra with known atmospheric parameters (Katz et al. 1998; Soubiran et al. 2003). Unlike Worthey et al. (1994) we chose not to calibrate the temperature of the giant stars on their spectral type as given by Ridgway et al. (1980) because this study is contested in several recent papers (Sekiguchi \& Fukugita 2000; Houdashelt et al. 2000). As a consequence, our estimated $T_{\text {eff }}$ for the giant stars is globally smaller by about $5 \%$ than the values given by Worthey et al. (1994). This will be discussed in Sect. 3.2.

The HR diagram coverage with the estimated parameters is extensive: $0.27<\log _{10}\left(g\left[\mathrm{~cm} \mathrm{~s}^{-2}\right]\right)<4.97$ and $3185 \mathrm{~K} \leq T_{\text {eff }} \leq 55200 \mathrm{~K}$, with $-3.21 \leq[\mathrm{Fe} / \mathrm{H}] \leq 1.62$. This coverage is illustrated in Fig. 1.

The up-to-date version of the library is available online ${ }^{3}$. Fully reduced spectra, as well as the estimated stellar parameters, are provided.

Since the number of spectra included in the ELODIE library has doubled since Prugniel \& Soubiran (2001a), we update here the comparison of the Lick indices measured on the stellar spectra with the reference indices from Worthey et al. (1994) for the 187 stars in common. Figure 2 illustrates this comparison for 16 indices, showing the generally good agreement between the two sets of spectra. However, a closer look reveals a general trend for our strongest indices to be weaker than the reference indices, which is expressed by the slopes being smaller than unity. To investigate this bias, we also compared our spectra to the Jones library (Leitherer et al. 1996; Jones 1998) for the 314 stars in common. This library has the advantage of being well calibrated in wavelength, as opposed to the Lick/IDS spectra of Worthey et al. (1994). When compared at the same resolution $(1.8 \AA)$, the two sets of spectra agree remarkably well.

\footnotetext{
2 And also http://www.obs.u-bordeaux1.fr/public/ astro/CSO/elodie_library.html

${ }^{3}$ http://www-obs.univ-lyon1.fr/ prugniel/soubiran/
}

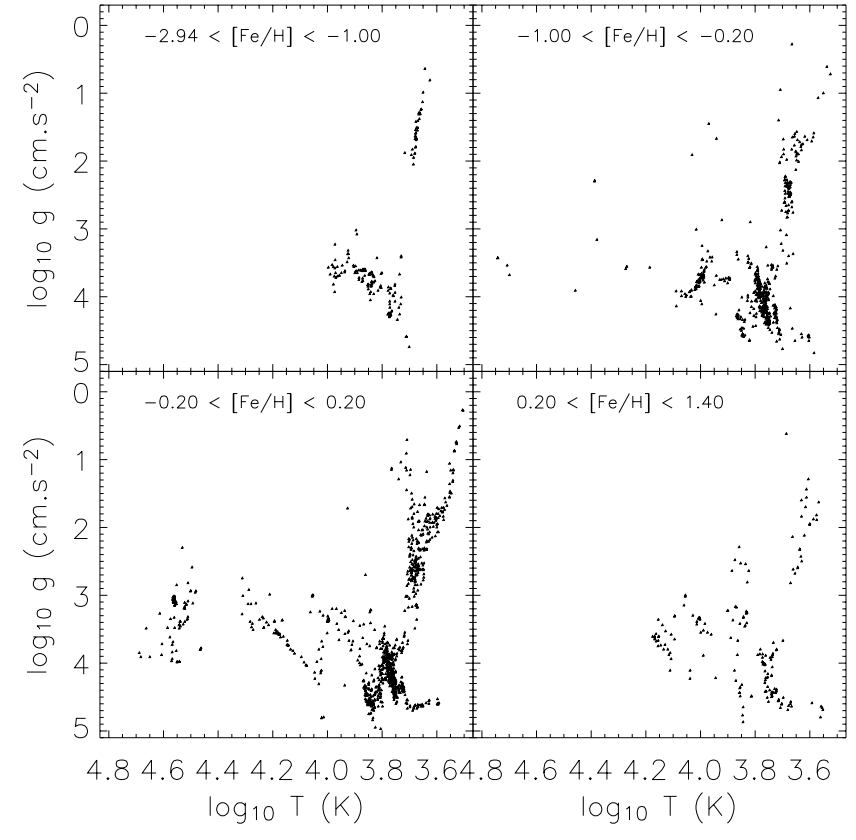

Fig. 1. Distribution in the $T_{\text {eff }}-\log _{10} g$ diagram of the 1503 stars in the ELODIE library in four bins of metallicity.

Table 1. Comparison of Lick indices measured on ELODIE stellar spectra with indices from other databases of stellar spectra, for stars in common. The indices for Worthey et al. (1994) were taken from the table published in their paper. The indices for the Jones library were measured by ourselves (see text). The values are the mean offset (ELODIE-other database), the slope of the linear regression and the dispersion $\sigma$ around it (see Fig. 2).

\begin{tabular}{|c|c|c|c|c|c|c|}
\hline \multirow{2}{*}{ Index } & \multicolumn{3}{|c|}{ Worthey et al. (1994) } & \multicolumn{3}{|c|}{ Jones library } \\
\hline & Offset & Slope & $\sigma$ & Offset & Slope & $\sigma$ \\
\hline Ca4227 & 0.038 & 0.772 & 0.151 & 0.023 & 0.890 & 0.074 \\
\hline G4300 & 0.025 & 0.910 & 0.333 & 0.094 & 0.942 & 0.191 \\
\hline $\mathrm{Fe} 4531$ & 0.017 & 0.849 & 0.245 & & & \\
\hline $\mathrm{Fe} 4668$ & 0.037 & 0.931 & 0.476 & & & \\
\hline $\mathrm{H} \beta$ & 0.022 & 0.947 & 0.140 & 0.002 & 0.958 & 0.069 \\
\hline Fe5015 & -0.136 & 0.884 & 0.358 & -0.001 & 0.966 & 0.163 \\
\hline $\operatorname{Mg} 1$ & -0.001 & 0.878 & 0.011 & -0.003 & 0.981 & 0.011 \\
\hline $\operatorname{Mg} 2$ & 0.001 & 0.918 & 0.009 & 0.000 & 0.963 & 0.007 \\
\hline $\mathrm{Mg}_{\mathrm{b}}$ & -0.037 & 0.877 & 0.204 & 0.019 & 0.996 & 0.070 \\
\hline $\mathrm{Fe} 5270$ & -0.033 & 0.978 & 0.252 & 0.012 & 0.991 & 0.057 \\
\hline Fe5335 & -0.017 & 0.974 & 0.214 & 0.003 & 0.947 & 0.061 \\
\hline $\mathrm{Fe} 5406$ & -0.003 & 0.937 & 0.203 & 0.008 & 0.994 & 0.047 \\
\hline Fe5782 & -0.011 & 0.745 & 0.120 & & & \\
\hline $\mathrm{NaD}$ & 0.073 & 0.895 & 0.314 & & & \\
\hline TiO1 & -0.001 & 0.889 & 0.010 & & & \\
\hline $\mathrm{TiO} 2$ & 0.001 & 0.909 & 0.007 & & & \\
\hline
\end{tabular}

Table 1 gives the quantitative comparison of these three libraries in terms of Lick indices. The indices of the Jones library were measured by ourselves when computing this table. The good agreement between ELODIE and Jones spectra is shown by the slopes being closer to unity and the smaller 

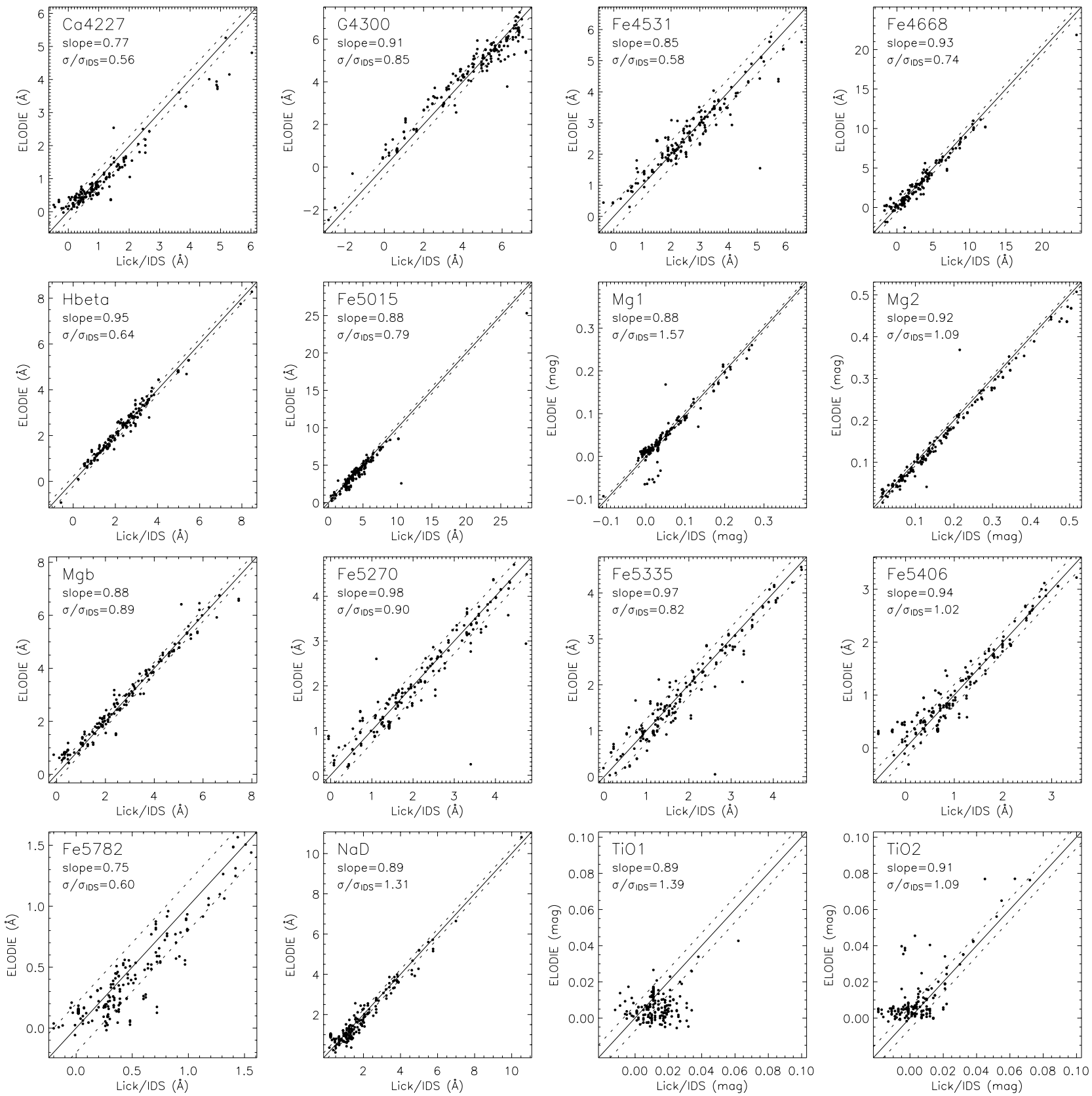

Fig. 2. Comparison of Lick indices measured on the ELODIE spectra with the reference Lick/IDS indices (Worthey et al. 1994) for 187 stars in common. The dotted lines are $y=x \pm \sigma_{\text {IDS }}$ where $\sigma_{\text {IDS }}$ is the uncertainty on the Lick/IDS indices. For each panel, we give the slope of the linear regression and the dispersion $\sigma$ around it. Ideally, $\sigma / \sigma_{\mathrm{IDS}} \simeq 1$.

dispersions $\sigma$. It is worth noticing that the indices published by Jones differ from the ones we measure from their spectra: they seem to have been artificially corrected in order to match the Worthey et al. (1994) indices by changing the overall slope. Our routine used to measure Lick indices was tested successfully on seven stars provided by G. Worthey. To measure the indices on the higher resolution spectra of the ELODIE or the Jones libraries, we use the wavelength-dependent resolution given in Worthey \& Ottaviani (1997).

The small bias observed between the measurements made on our library and the Jones library is probably due to the imperfect calibration of the spectra in either library. The reason for the discrepancy between our measurements and the
Lick/IDS ones is still unclear, but it might be attributed to a systematic effect in the wavelength scale of the Lick library.

In the following, we do not correct any of our indices for this very small bias. Section 3.2 will show that its consequences on SSPs are usually small, although they may become important when red giants dominate the spectra.

To use the set of ELODIE spectra as an input stellar library to PEGASE, the spectra were interpolated on the BaSeL (Lejeune et al. 1997) grid of parameters $\left(T_{\mathrm{eff}}, \log _{10} g,[\mathrm{Fe} / \mathrm{H}]\right)$ with piecewise polynomials similar to the Lick fitting functions (Worthey 1994), in various regions of the HR diagram. The interpolated spectra were then normalized to match the mean BaSeL fluxes in the band 5500-6000 A. The detailed 
description of the interpolator, as well as the comparison of the BaSeL library with the interpolated stellar library, are described on a web site ${ }^{4}$ and will be published in a forthcoming paper (Prugniel \& Soubiran 2004).

The range of stellar parameters in the BaSeL library being much larger than the range of parameters covered by the ELODIE library, we chose not to use the extrapolated spectra of the interpolated library. To exclude the extrapolated spectra, we set a minimum level for the density of real stellar spectra at each point of the BaSeL grid. The resulting interpolated library then contains fewer spectra than the BaSeL library (2690 instead of 4422), but each of its spectra is reliable. A minor modification in the PÉGASE algorithm was necessary to use this new grid.

\subsection{SSPs at high spectral resolution}

Figure 3 presents a prediction of a 10 Gyr-old high resolution SSP spectrum at solar metallicity.

The labeled grey areas in the plot identify the passbands of some Lick indices (Worthey et al. 1994). We can notice that each passband includes many lines of various chemical elements. More precise line identifications and their evolution are presented in the next section. To highlight the spectral resolution improvement, we have overplotted the same SSP computed with PEGASE. 2 using the BaSeL library.

It is worth noting that the adopted evolutionary tracks are parametrized by their total metallicity $Z$, while the stellar library is labeled by the iron abundance $[\mathrm{Fe} / \mathrm{H}]$. In the present work, we assume the relation $\log _{10}(\mathrm{Z} / 0.02)=[\mathrm{Fe} / \mathrm{H}]$. The possible correlation between metallicity and relative abundances of $\alpha$-elements in the spectral library, due to the selection of stars from the solar neighborhood, will be invoked in the following to explain some of the differences with other models.

\subsection{The spectra around specific stellar lines}

Figure 6 presents the normalized spectral distributions of SSPs centered on the main stellar lines $\left(\mathrm{H} \delta, \mathrm{H} \gamma, \mathrm{H} \beta, \mathrm{Mg}_{\mathrm{b}}, \mathrm{Fe} \lambda 5270\right.$, $\mathrm{Fe} \lambda 5335, \mathrm{NaD})$. The qualitative effects of age and metallicity can be assessed: in the left column, all SSPs have solar metallicity and their ages are at 1, 4, $13 \mathrm{Gyr}$; in the right column, all SSPs are $10 \mathrm{Gyr}$-old and their metallicities are $[\mathrm{Fe} / \mathrm{H}]=-1.7$, $-0.4,0.4$. Lick index passbands are shown with vertical lines and the grey areas correspond to the pseudo-continuum passbands. The widths of the Lick passbands contrast with the narrow profiles of the stellar lines, outlining the possibility of refined indices.

\subsection{The library of SSPS}

Further examples of synthetic SSPs spectra at the resolutions of PEGASE.2 and PEGASE-HR are shown in Fig. 5. For clarity, PEGASE. 2 spectra are offset vertically. The qualitative agreement in the energy distribution and the broad features is very

\footnotetext{
${ }^{4}$ http://www.obs.u-bordeaux 1.fr/public/astro/CSO/ elodie_library.html
}

satisfactory. A quantitative comparison is provided below. Note that PEGASE-HR and PEGASE. 2 are based on the same algorithm, so that the evolution of global parameters (metallicity, mass of gas, stellar mass and all outputs independent of the library) is unchanged.

\section{Consistency of the PEGASE-HR outputs}

\subsection{Quantitative comparison to PEGASE.2: Luminosities and colors}

Figure 7 presents a comparison of the integrated fluxes predicted by PEGASE-HR and by PEGASE. 2 through the quantity

$$
\delta F=-2.5 \times \log _{10}\left(\frac{\int_{\lambda=4200 \AA}^{6700 \AA} F_{\lambda}^{\mathrm{PEGASE}-\mathrm{HR}} \mathrm{d} \lambda}{\int_{\lambda=4200 \AA}^{6700 \AA} F_{\lambda}^{\mathrm{PEGASE} .2} \mathrm{~d} \lambda}\right),
$$

for SSPs of various ages (1 Myr to $20 \mathrm{Gyr}$ ) and metallicities (from $[\mathrm{Fe} / \mathrm{H}]=-1.7$ to $[\mathrm{Fe} / \mathrm{H}]=0.7$ ). Absolute differences for ages greater than $10 \mathrm{Myr}$ and $[\mathrm{Fe} / \mathrm{H}]>-1.7$ are always smaller than 0.04 mag.

Figure 8 compares the slopes of the energy distributions produced by the two versions of the code. The slope is measured with a color index, based on filters with rectangular passbands spanning 4300-4600 A ("blue" band) and 6400-6700 ̊ ("red" band). Color differences between the two models, over the whole range of metallicities and ages greater than $10 \mathrm{Myr}$, are lower than $0.12 \mathrm{mag}$. Once more, the largest disagreements are confined to the youngest ages and lowest metallicities.

Overall, the colors and fluxes of PEGASE-HR are consistent with those of PEGASE. 2 for ages greater than $10 \mathrm{Myr}$ and $[\mathrm{Fe} / \mathrm{H}]>-1.7$. This result confirms that the BaSeL and ELODIE libraries are very similar at low resolution, at least over the range of parameters in which stellar spectra contribute significantly to the optical light of a galaxy. Such an agreement was not obvious a priori since the two libraries are constructed with very different methods: the former is based on theoretical spectra, color-corrected to fit observed stellar continua, whereas the latter is purely empirical. As a consequence, the low-resolution extended SEDs predicted by PEGASE. 2 and the high-resolution spectra of PEGASE-HR can be used together to refine SED studies.

\subsection{Comparison with Lick indices for SSPS}

Before studying lines at high resolution, we first want to check that our predictions at medium resolution are consistent with those of other models.

The traditional approach for the computation of Lick indices of SSPs (e.g. Worthey \& Ottaviani 1997; Bressan et al. 1996) is based on a library of indices of individual stars and on analytic representations of this set of data (the fitting functions). Thanks to the resolution of the ELODIE spectra, our approach is more direct: we compute the synthetic spectrum of a galaxy or SSP, degrade the spectral resolution to the one of 

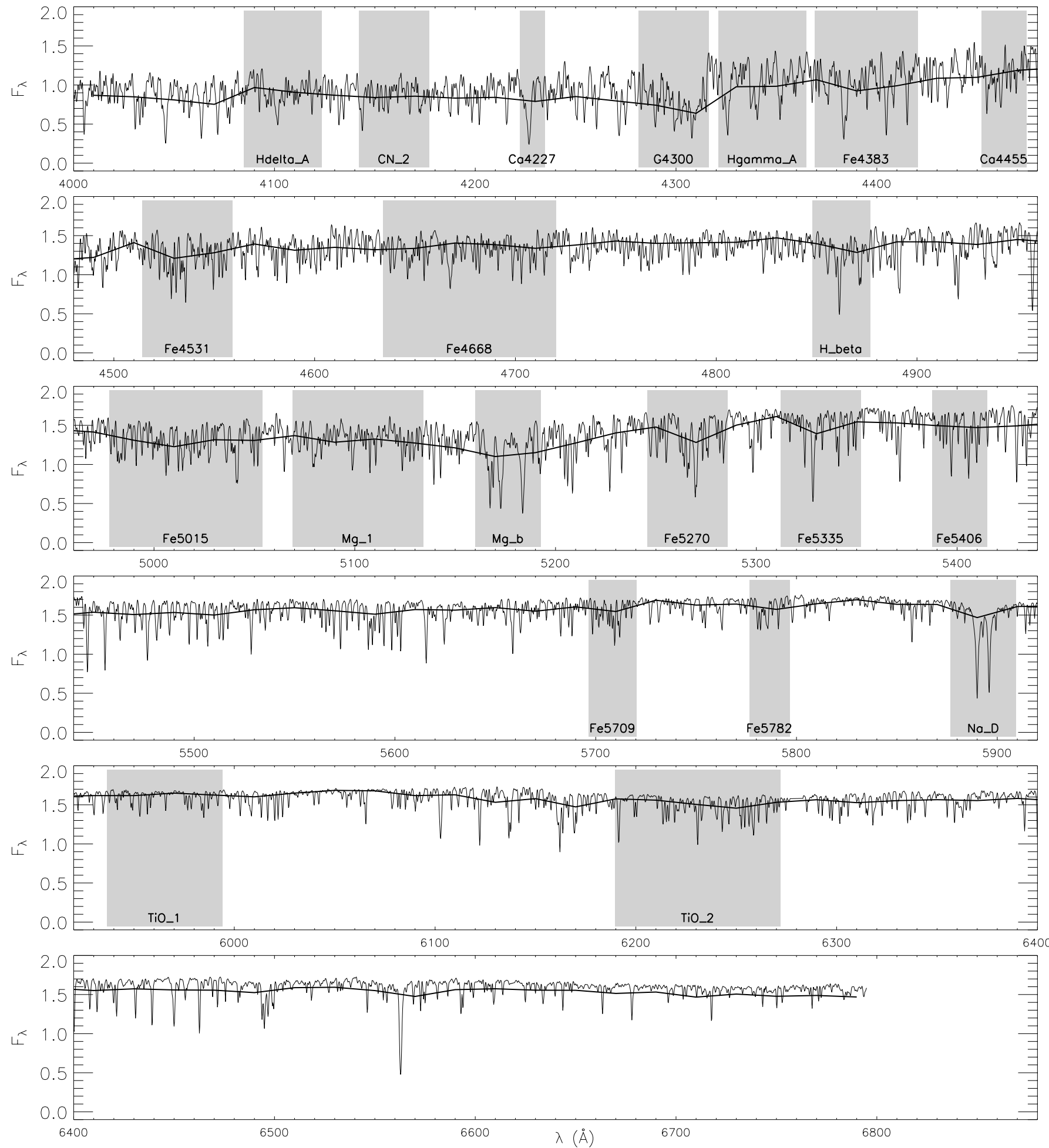

Fig. 3. PEGASE-HR high-resolution spectrum of a 10 Gyr-old SSP of solar metallicity compared to the low resolution spectrum from PEGASE.2. The flux is given in units of $10^{-5} L_{\odot} \AA^{-1} M_{\odot}^{-1}$, where $L_{\odot}=3.826 \times 10^{33} \mathrm{erg} \mathrm{s}^{-1}$. Grey areas correspond to the passbands of some Lick indices.

the Lick index definitions (8 to 11 A depending on wavelength, as described by Worthey \& Ottaviani 1997), and then measure the indices directly on the smoothed spectrum. This allows us to easily predict indices for any complex evolutionary scenario. In the following, we compare our results for SSPs with those of other groups.

\subsubsection{Metal-sensitive indices}

The left hand side of Fig. 9 shows the variations with age of the metal-sensitive Lick indices Fe5270, Fe5335, $\mathrm{Mg}_{\mathrm{b}}$ and $\mathrm{NaD}$, as computed with PEGASE-HR, by Bressan et al. (1996), by Thomas et al. (2003), and by Bruzual \& Charlot (2003). 


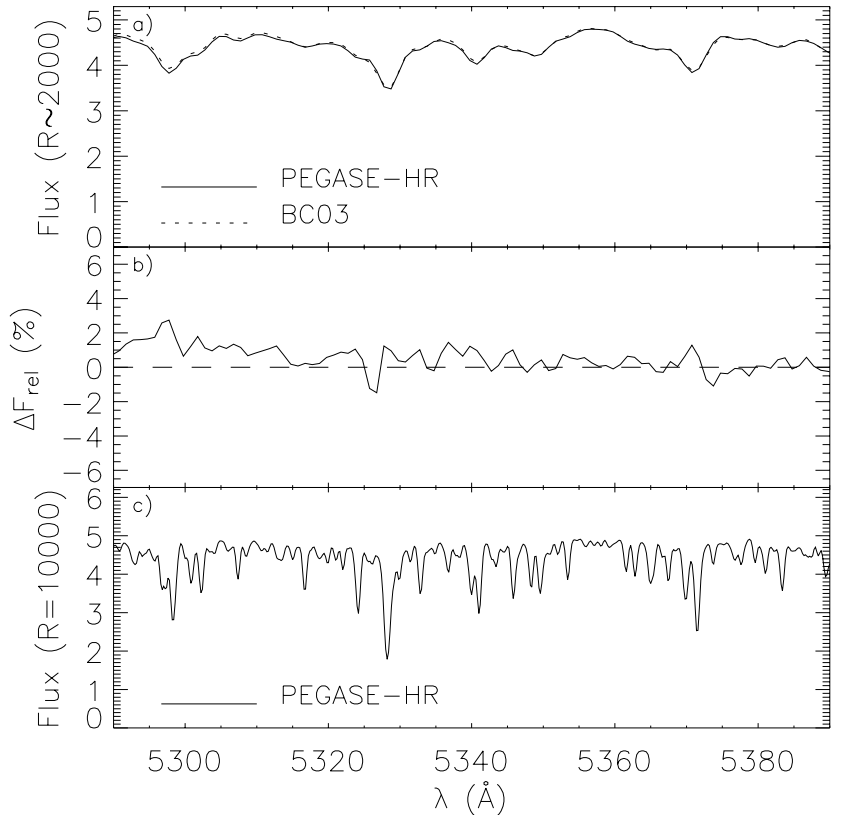

Fig. 4. Comparison of a portion of a smoothed PEGASE-HR $\operatorname{SSP}$ spectrum $\left(Z_{\odot}, 3 \mathrm{Gyr}\right)$ with the model of Bruzual \& Charlot (2003). The IMF is common to both models (Salpeter 1955). a) Both models overplotted, at the same low resolution $R \simeq 2000$. The flux is given in units of $10^{-5} L_{\odot} \AA^{-1} M_{\odot}^{-1}$. b) Relative difference of the fluxes at low resolution $(R \simeq 2000)$. c) The original high resolution PEGASE-HR SSP spectrum $(R=10000)$.

The comparisons are shown at various metallicities. Thomas et al. (2003) provide results for various relative abundances of the $\alpha$-elements, and we have plotted those for $[\alpha / \mathrm{Fe}]=0$. The Salpeter (1955) initial mass function (IMF) is used in the three models (power law index $x=-1.35$ ). The lower and upper stellar masses are adapted for each comparison.

PEGASE-HR, Bressan et al. (1996) and Bruzual \& Charlot (2003) all use the Padova stellar tracks in this comparison, while Thomas et al. (2003) use what we will call the "Cassisi tracks", a compilation from Cassisi et al. (1997), Bono et al. (1997), Maraston (1998), Salasnich et al. (2000). Moreover, both Thomas et al. (2003) and Bressan et al. (1996) use "fitting functions" to derive their synthetic indices, contrarily to us and to Bruzual \& Charlot (2003).

The agreement of the iron indices between the three models is quite good, except for the youngest SSPs at the lowest metallicities. This shows that ELODIE spectra and the iron index fitting functions of Worthey et al. (1994) are compatible over the range of parameters relevant for the synthesis of stellar populations, as expected from the direct comparison performed by Prugniel \& Soubiran (2001a). As stated in Maraston et al. (2003), the Cassisi tracks should lead to slightly lower values of the indices. This is what we observe, especially for iron indices. The discrepancy with Bruzual \& Charlot (2003) at super-solar metallicity will be explained below.

The situation for the $\mathrm{Mg}_{\mathrm{b}}$ index is more complex, since magnesium is an $\alpha$-element. Both the ELODIE library and the Lick fitting functions rely on an empirical stellar data set, in which one should expect the anti-correlation between $[\mathrm{Fe} / \mathrm{H}]$ and $[\alpha / \mathrm{Fe}]$ characterizing the solar neighborhood

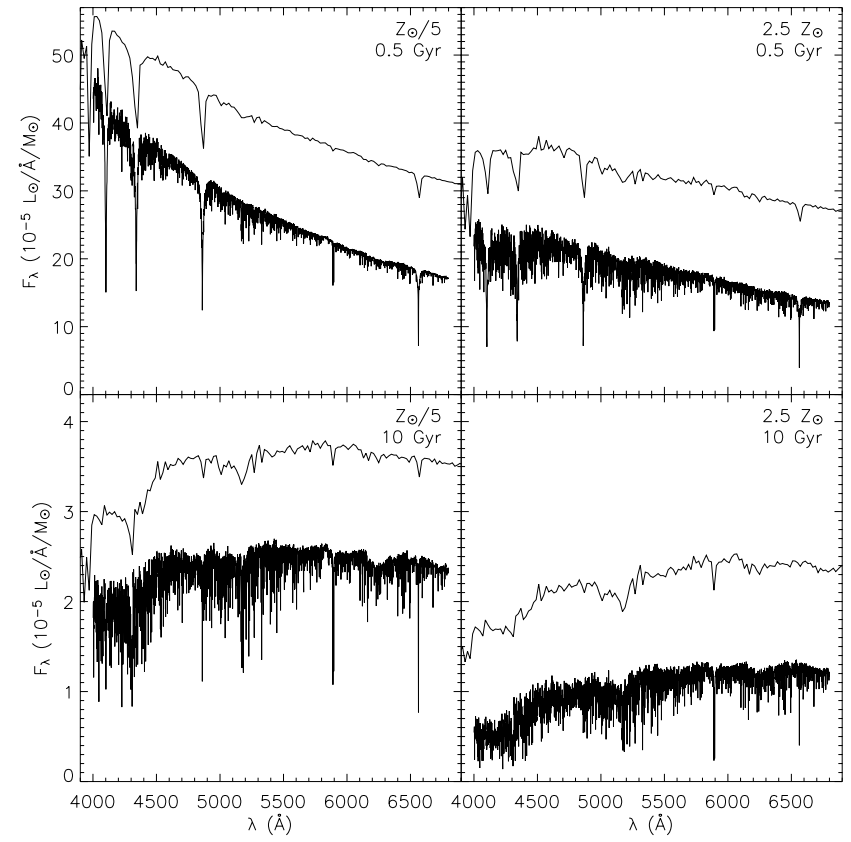

Fig. 5. Comparison of PEGASE.2 and PEGASE-HR spectra of SSPs, at metallicities $2.5 \times Z_{\odot}$ and $Z_{\odot} / 5$, and ages $0.5 \mathrm{Gyr}$ and $10 \mathrm{Gyr}$. The PEGASE. 2 spectra are plotted with a vertical offset (a constant in each panel) for clarity.

(Fuhrmann 1998; Fulbright 2002). Thomas et al. (2003) have included a correction for the local anti-correlation between $[\mathrm{Fe} / \mathrm{H}]$ and $[\alpha / \mathrm{Fe}]$ in their sub-solar computations. At solar metallicity, where no such correction is required, their predictions and those of PEGASE-HR agree very well. As expected, PEGASE-HR produces a higher $\mathrm{Mg}_{\mathrm{b}}$ index at sub-solar metallicities. We have observed that our predictions match better the values given by Thomas et al. (2003) for $[\alpha / \mathrm{Fe}]=0.3$.

The differences between the $\mathrm{Mg}_{\mathrm{b}}$ predictions of PEGASE-HR and those of Bressan et al. (1996), which are significant at super-solar metallicities, can only result from selection effects in the underlying stellar samples or in peculiarities of the interpolations in regions of the HR diagram where only few stars are available. Although the differences in stellar samples are probably the main source of disagreement between the models at sub-solar metallicity, the latter option is likely to explain the strong difference observed at $[\mathrm{Fe} / \mathrm{H}]=0.4$, as demonstrated below.

We have compared the $\mathrm{Mg}_{\mathrm{b}}$ indices measured on the ELODIE spectra (after interpolation onto the grid of fundamental parameters used in the population synthesis code) with those obtained for the same grid of parameters from the fitting functions of Worthey et al. (1994). The average offset is negligible, as can be expected from Prugniel \& Soubiran (2001a) and Fig. 2. However, the indices measured on ELODIE spectra of non-giant stars $\left(\mathrm{Mg}_{\mathrm{b}}<6\right)$ have a slight tendency to have lower values than those derived from fitting functions for strong lines, and higher values for weak lines. This effect, also present for the individual stars (see Fig. 2), is of the order of a few tenths of an Ångström, i.e. not sufficient to explain the differences observed for $\mathrm{Mg}_{\mathrm{b}}$ in Fig. 9 between PEGASE-HR and Bressan et al. (1996). More important is that the $\mathrm{Mg}_{\mathrm{b}}$ fitting functions 

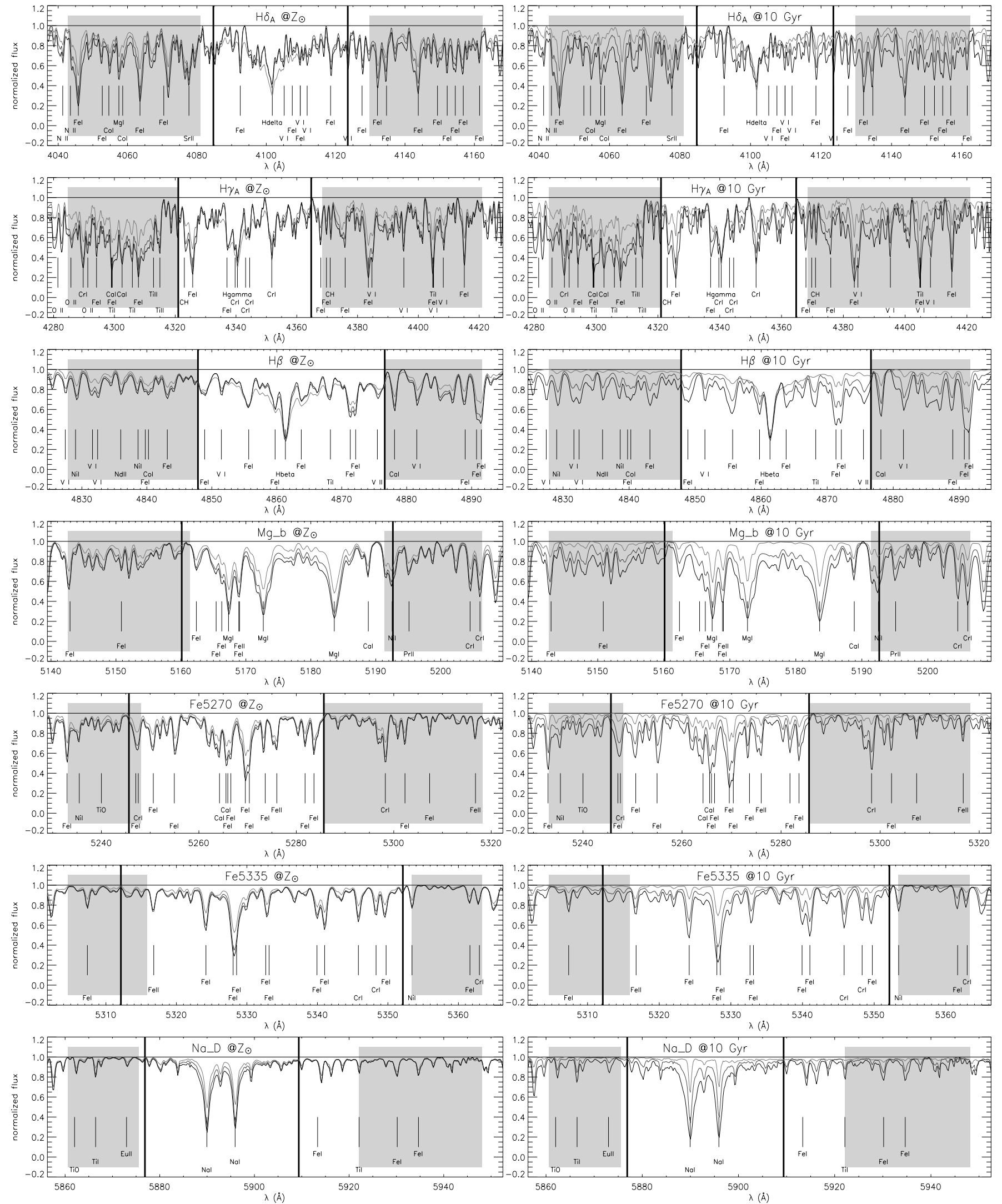

Fig. 6. Evolution of main stellar lines and continua for an SSP as a function of age (ages $=1,4,13 \mathrm{Gyr}$, from light gray to black) at $Z_{\odot}($ left) and metallicity $([\mathrm{Fe} / \mathrm{H}]=-1.7,-0.4,0.4$, from gray to dark) at $10 \mathrm{Gyr}($ right $)$. Line identifications are from the revised ILLSS catalogue (Coluzzi 1999). Grey areas show the blue and red pseudo-continua of the Lick indices and solid vertical lines delimit their central passband. In some cases $\left(\mathrm{H} \gamma_{\mathrm{A}}, \mathrm{H} \delta_{\mathrm{A}}\right)$, significant evolution with age is observed within the pseudo-continua (see text). 


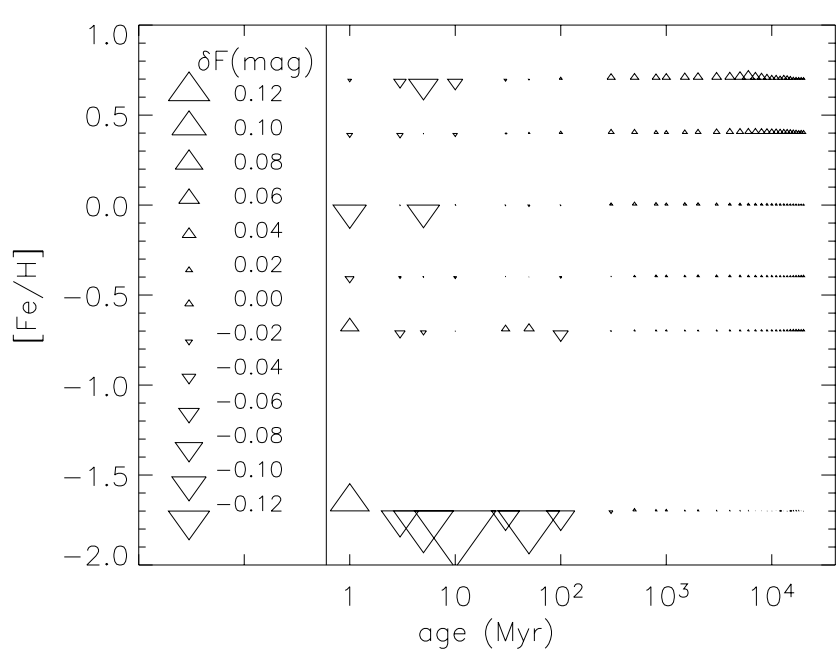

Fig. 7. Comparison of the integrated optical flux predicted by PEGASE. 2 and PEGASE-HR for SSPs using $\delta F$ (4200-6700 ̊). The size of the symbols scales with magnitude difference (see text in Sect. 3.1 for details).

rise dramatically with decreasing temperatures for low gravity red giant stars $\left(T_{\text {eff }}<3800 \mathrm{~K}\right)$. When using the Padova stellar evolution tracks, these stars contribute significantly to the optical emission of old metal-rich populations. Very few such stars were present in the library used by Worthey et al. (1994), all of them with sub-solar metallicity, and their $\mathrm{Mg}_{\mathrm{b}}$ indices are high indeed (values above $15 \AA$, up to $18.5 \AA$ ). The $\mathrm{Mb}_{\mathrm{b}}$ indices of the coolest super-solar giants in the ELODIE library are lower by up to several $\AA$ : the strongest $\mathrm{Mg}_{\mathrm{b}}$ index for a giant is $\mathrm{Mg}_{\mathrm{b}}=14.3 \AA$. Also, there are only two giant stars in the ELODIE library with $[\mathrm{Fe} / \mathrm{H}]>0$ and $\mathrm{Mg}_{\mathrm{b}}>7 \AA$ (HD 18191 and HD 169931). The interpolation of the stellar spectra is very hazardous with so few stars in this range of metallicity, effective temperature and surface gravity. Therefore, one should not be surprised to observe strong differences in the indices of $\alpha$-elements for old, super-solar stellar populations.

Moreover, a comparison of our estimated effective temperatures with the values published in Worthey et al. (1994) for the stars in common shows that the calibration of $T_{\text {eff }}$ for the cool giant stars is uncertain by about $150 \mathrm{~K}(5 \%)$ which could also explain some of the differences between the models at super-solar metallicities. The effect of this uncertainty on the predicted indices at $[\mathrm{Fe} / \mathrm{H}]=0.4$ is comparable to the discrepancy between the models: Fig. 10 shows four indices of $\mathrm{a}[\mathrm{Fe} / \mathrm{H}]=0.4 \mathrm{SSP}$ obtained by shifting artificially the temperatures of the stellar tracks by $\pm 5 \%$ (shaded region). The other models at this metallicity (Bruzual \& Charlot 2003; Bressan et al. 1996) predict values compatible with this uncertainty. However, this shaded region probably overestimates the error due to the uncertainty in stellar effective temperatures: the error in $T_{\text {eff }}$ for the dwarf main sequence stars is smaller than $5 \%$. At solar or sub-solar metallicities, the contribution of the giant stars to the spectrum of an SSP is smaller. The uncertainty in $T_{\text {eff }}$ then becomes negligible in comparison to the uncertainty in the stellar tracks.

The index $\mathrm{NaD}$ is in quite good agreement with the other models, which is remarkable because this index is

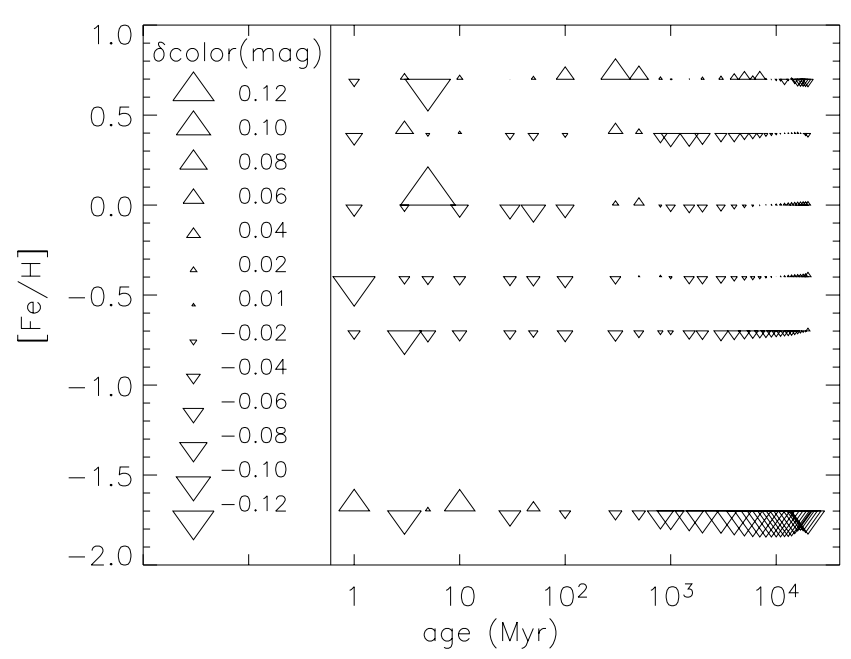

Fig. 8. Comparison of the optical colors predicted by PEGASE-HR and PEGASE. 2 for SSPs. The adopted color compares the fluxes integrated over $4300-4600 \AA$ in the blue and $6400-6700 \AA$ in the red. The size of the triangles scales with the color difference expressed in magnitudes ( $\delta$ color) between the two models. Head-up triangles are plotted when PEGASE-HR produces a redder slope than PEGASE.2.

contaminated by many telluric lines. We took great care to remove these lines from the high resolution stellar spectra. The non-monotonic evolution of the $\mathrm{NaD}$ index with metallicity for old SSPs in the predictions of Bruzual \& Charlot (2003) reflects the difficulty to model this index correctly.

\subsubsection{Hydrogenic indices, age tracers}

Balmer lines are generally considered to be excellent age tracers. $\mathrm{H} \alpha$ and $\mathrm{H} \beta$ are often strong absorption lines, but they may be filled in by circumstellar and interstellar emission. Thus, they provide less reliable age diagnostics than $\mathrm{H} \gamma$ and $\mathrm{H} \delta$ which are much less affected.

To analyze stellar populations, Worthey \& Ottaviani (1997) defined Lick indices around easily measurable Balmer lines: $\mathrm{H} \beta_{\mathrm{A}}, \mathrm{H} \gamma_{\mathrm{A}}$ and $\mathrm{H} \delta_{\mathrm{A}}$ for $\mathrm{A}$ stars with broadened wings, and $\mathrm{H} \beta_{F}, \mathrm{H} \gamma_{F}$ and $\mathrm{H} \delta_{F}$ for later types, with narrower Balmer lines. They use the Revised Yale isochrones (Green et al. 1987) and Vandenberg (1985) isochrones extrapolated, when necessary, to some parts of the HR diagram. The IMF adopted by them has a Salpeter (1955) slope, with $M_{\min }=0.1 M_{\odot}$ and $M_{\max }=2 M_{\odot}$ (they model old populations only). Figure 9 (right hand side) shows an overall satisfactory agreement between PEGASE-HR, Worthey \& Ottaviani (1997), Thomas et al. (2003) and Bruzual \& Charlot (2003) for metallicities $[\mathrm{Fe} / \mathrm{H}]>-1.00$. However, we are also in rather good agreement with Bruzual \& Charlot (2003) for $[\mathrm{Fe} / \mathrm{H}]=-1.7$. We believe that some of the discrepancies in the predictions of Lick indices for Balmer lines at low metallicity are due to different treatments of the helium-burning stars in the stellar tracks (Charlot et al. 1996; Yi 2003). This is illustrated in the right panels of Fig. 9 where the Bruzual \& Charlot (2003) predictions for solar metallicity are given with the Padova tracks, but also with the Geneva (Schaller et al. 1992; Charbonnel et al. 1996) tracks. As for metal-sensitive indices, many of the 

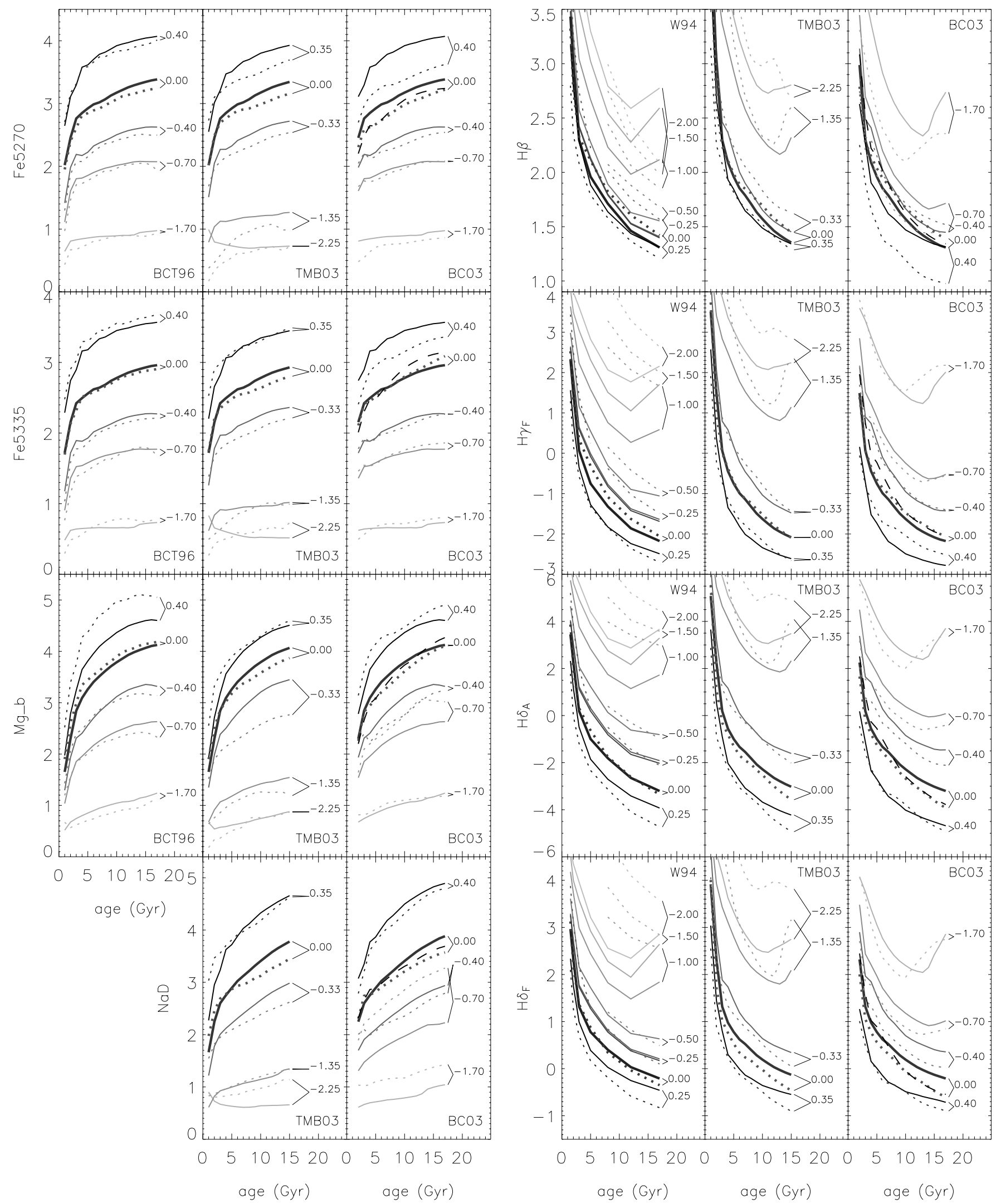

Fig. 9. PEGASE-HR Lick indices (solid lines) for SSPs compared to models (dotted lines) of Worthey \& Ottaviani (1997, W95), Bressan et al. (1996, BCT96), Thomas et al. (2003, TMB03) and Bruzual \& Charlot (2003, BC03). The figures on the right hand side of each panel represent the quantity $[\mathrm{Fe} / \mathrm{H}]$. For BC03 only, the dashed line shows the indices computed with the Geneva tracks instead of the Padova 1994 tracks, for solar metallicity. 

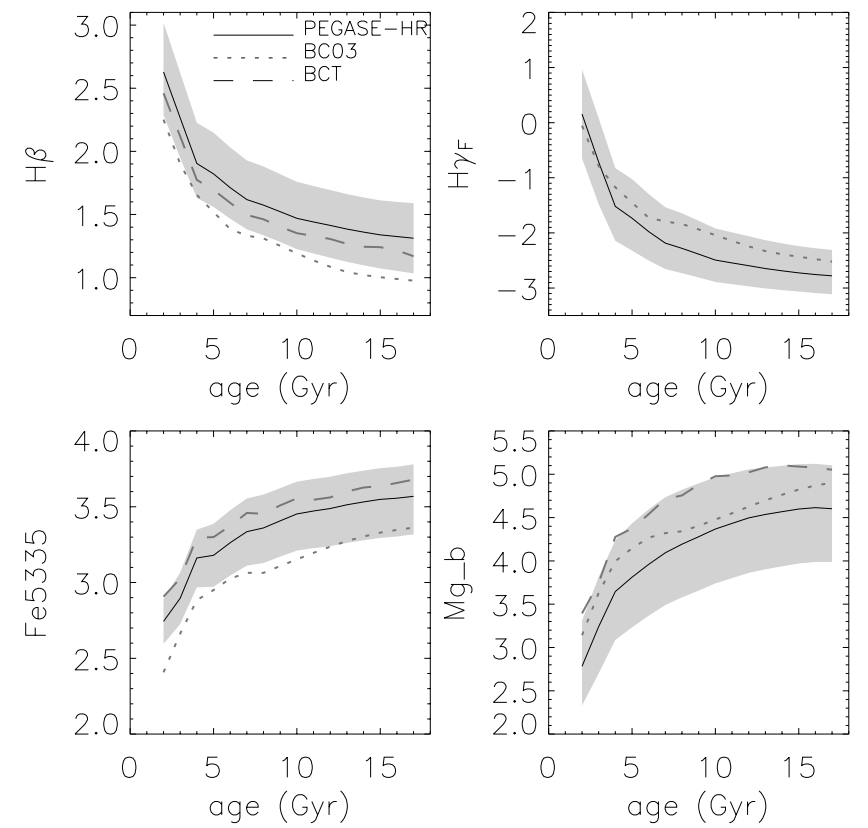

Fig. 10. Lick indices for $[\mathrm{Fe} / \mathrm{H}]=0.4$ SSPs. The indices of this work (solid line), Bruzual \& Charlot (2003) (dotted line) and Bressan et al. (1996) (dashed line) are shown. The shaded region shows the uncertainty in the indices associated to the uncertainty in the effective temperature of the giant stars (see text for details).

discrepancies in the predictions of the Balmer Lick indices, in particular at super-solar metallicities, are likely due to differences in the stars used to build the stellar libraries and in their estimated effective temperatures.

\section{Synthesis of evolved populations}

\subsection{Globular clusters}

Predictions of spectral feature strengths in SSP spectra can be directly compared to observational data of coeval stellar populations, as found in globular clusters. The data used here were obtained by Bica \& Alloin (1986a), Huchra et al. (1996) and Trager et al. (1998).

\section{Equivalent width of the $\mathrm{H} \beta$ line}

Equivalent widths $(E W)$ of the $\mathrm{H} \beta$ line were measured as a function of age and metallicity for a sample of 63 observed star clusters in the Galaxy, the LMC, and the SMC by Bica \& Alloin (1986a,b). We predict the evolution of the $\mathrm{H} \beta E W$ with PEGASE-HR, with the same spectral resolution (12 $\AA$, Gaussian smoothing) and the same $E W$ definition:

$E W=\int_{W}\left[1-F(\lambda) / F_{\mathrm{c}}(\lambda)\right] \mathrm{d} \lambda$

where $F_{\mathrm{c}}$ is the continuum in the middle of the window $W$. The continuum is the upper envelope of the spectrum: maximal fluxes around the points $\lambda \lambda=4570,5340,6630 \AA( \pm 20 \AA)$ are connected by straight lines. $E W$ is given in $\AA$.

Figure 11 plots the binned data compared to the PEGASE-HR sequences of $\mathrm{H} \beta$ equivalent widths for various

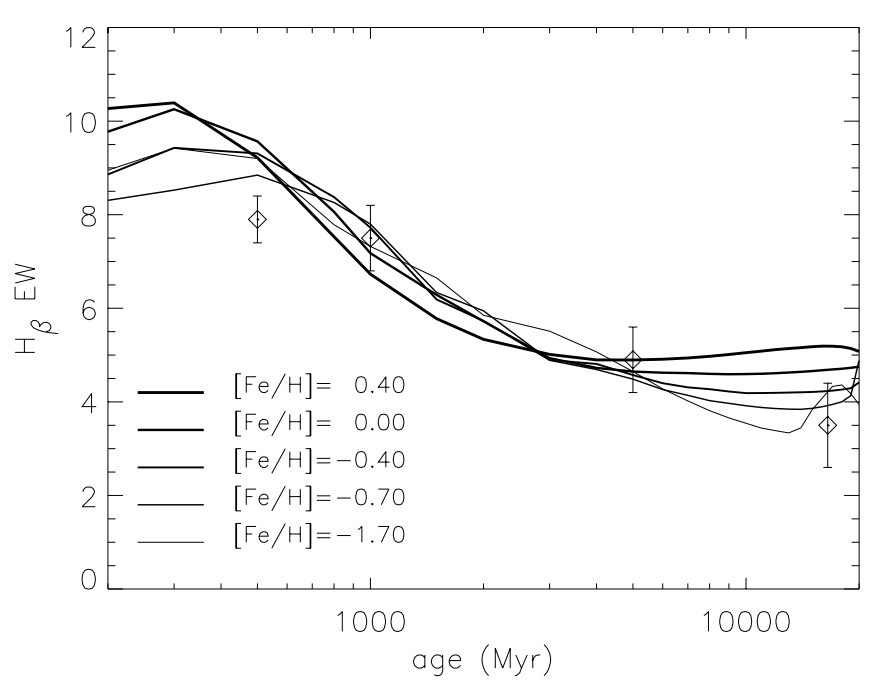

Fig. 11. Evolution sequences with age of the $\mathrm{H} \beta$ equivalent width from PEGASE-HR for various metallicities (solid lines) compared to the binned globular cluster data (Bica \& Alloin 1986a,b).

metallicities $([\mathrm{Fe} / \mathrm{H}]=-1.7,-0.7,-0.4,0 ., 0.4)$. Note that the isochrones used by Bica \& Alloin (1986b) to estimate ages differ from the ones used here, allowing no very precise age comparison.

The models agree with the data at ages roughly between 1 and 10 Gyr. At younger ages, emission lines might partly fill in absorption lines in the observations, and ongoing star formation may rule out the model of the strictly instantaneous $(<1 \mathrm{Myr})$ burst described by an SSP. At the oldest ages, the metal deficiency of globular clusters is extreme, and we reach the regime in which our interpolated spectral library suffers from larger uncertainties.

\section{The iron- $\mathrm{H} \beta$ diagram}

Among the 13 indices measured in 193 extragalactic globular clusters by Huchra et al. (1996), we select the index Fe5270 as a representative metallicity tracer and $\mathrm{H} \beta$ as the best available age tracer (see also Table 3). The definitions of indices in Huchra et al. (1996) differ from the Lick ones:

$$
I=-2.5 \log \left[\frac{2 F_{I}}{\left(F_{C 1}+F_{C 2}\right)}\right]
$$

where $F_{I}, F_{C 1}$ and $F_{C 2}$ are mean fluxes in the passband, blue and red continua respectively. The passbands are also different from the Lick ones. In Fig. 12, the grid of PEGASE-HR models (with ages $=0.3-14 \mathrm{Gyr}$ and $[\mathrm{Fe} / \mathrm{H}]=-1.7$ to 0.4 ) is compared to the data sample. The grid encompasses a wide range of data, when taking into account the large observational error bar. However, several points (mostly M 31 clusters) are outside the theoretical grid. Assuming that the behavior of the indices of Huchra et al. (1996) is comparable to that of the corresponding Lick indices, the offset in $\mathrm{H} \beta$ for the lowest metallicity clusters is consistent with the uncertainties already mentioned for this regime. For M 33 clusters, Brodie \& Huchra (1991) give estimations of the individual metallicities. Overall, we find a rather good agreement between measured metallicities of clusters and 


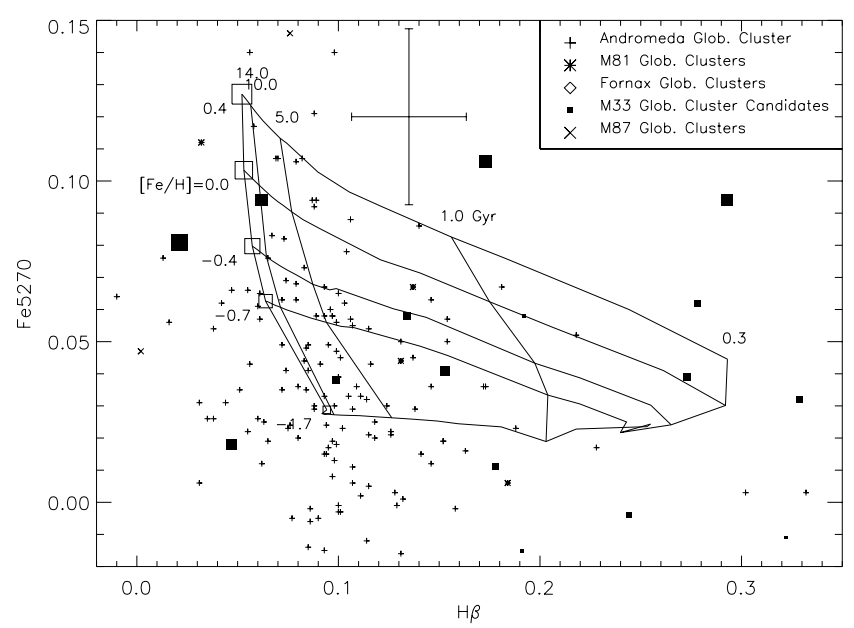

Fig. 12. Fe 5270 vs. $\mathrm{H} \beta$ indices for 193 extragalactic globular clusters (Huchra et al. 1996). Solid lines are PEGASE-HR SSP models for several ages and metallicities, as labeled on the sequences. The typical error bar for the data is plotted above the grid. For M 33 globular clusters, the size of the full squares indicates $[\mathrm{Fe} / \mathrm{H}]$ (as determined by Huchra et al. 1996), following the $[\mathrm{Fe} / \mathrm{H}]$ scale given by the empty squares plotted on the $14 \mathrm{Gyr}$ isochrone.

the grid. This agreement is illustrated by the size of the squares in the plot (filled squares for M 33 data and empty squares for the model predictions).

More recently, some precise measurements of Lick indices were made on globular clusters of the Milky way and M 31 and were compiled by Trager et al. (1998). The measurement error is smaller for these data, and enables a more precise determination of ages. Figure 13 shows the observed values of Fe5270 and $\mathrm{H} \beta$ together with our synthetic grid for these indices. It appears that the locus of the globular clusters in the grid is approximately the same as for the extragalactic clusters of Fig. 12. However, the ages are determined much more precisely and they span a narrower range (3-20 Gyr) with mainly sub-solar metallicities.

\subsection{Elliptical galaxies}

Elliptical galaxies are often modeled as purely coeval stellar populations since the bulk of their stellar population formed within a very short timescale (less than 1 Gyr). We hereafter prefer to use the typical scenarios of the model PEGASE (Fioc \& Rocca-Volmerange 1997) including infall and galactic winds. The star formation rate is proportional to the mass of gas, with respective timescales of 1 Myr for starburst, less than 1 Gyr for elliptical and up to $10 \mathrm{Gyr}$ for spiral galaxies.

Figure 14 shows the predictions of the main Lick indices with our three scenarios (spiral, elliptical, and instantaneous burst, as defined in Le Borgne \& Rocca-Volmerange 2002) compared to the same indices measured on local elliptical galaxies (Trager et al. 2000). The assumed galaxy age is $13 \pm 0.5 \mathrm{Gyr}$, but the indices are not very sensitive to age in this range. $\mathrm{Mg}_{\mathrm{b}}$ predictions are systematically low, which is

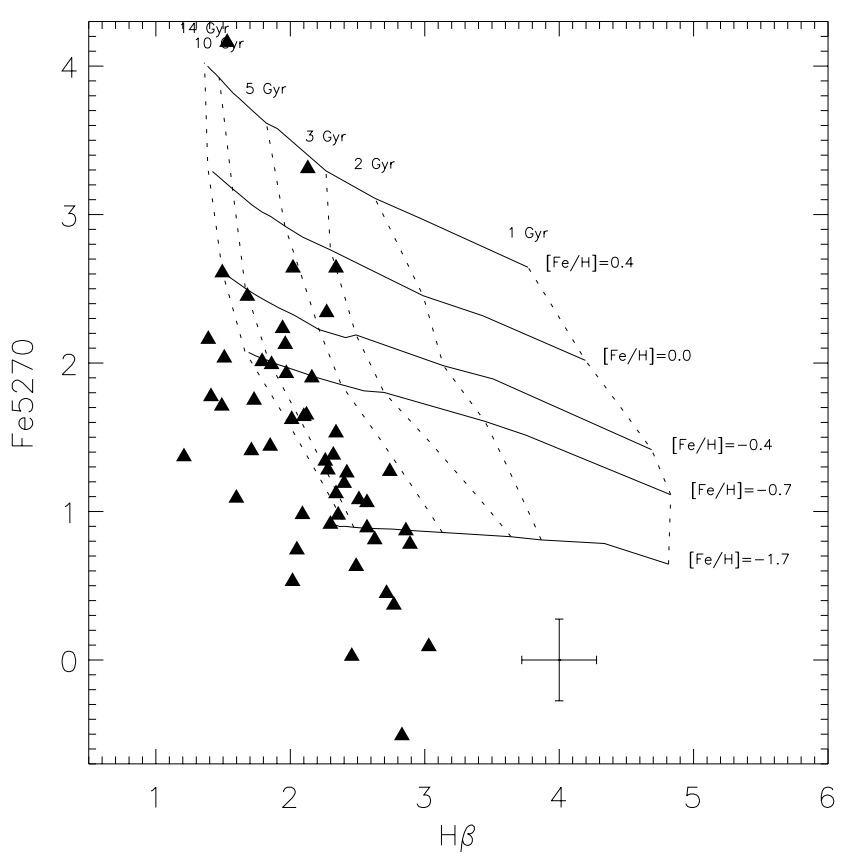

Fig. 13. Fe 5270 vs. $\mathrm{H} \beta$ indices for 50 globular clusters of the Milky Way and M 31 (Trager et al. 1998). The grid corresponds to PEGASE-HR SSP models, with iso-age sequences (dotted lines) and iso- $[\mathrm{Fe} / \mathrm{H}]$ sequences (solid lines). The median error bar for the data is plotted below the grid.

consistent with the known over-abundance of $\alpha$-elements in elliptical galaxies.

\section{A systematic search for new narrow indices}

Lick indices are considered, to a certain extent, to be good estimators for age $\left(\mathrm{H} \beta\right.$ index) or metallicity ( $\mathrm{Fe}$ or $\mathrm{Mg}_{\mathrm{b}}$ indices). Yet these indices are defined for medium resolution $(F W H M \sim 8 \AA)$ : many absorption lines, from various elements, are included in each passband. The full resolution power of PEGASE-HR makes it possible to build new narrow indices for a better estimation of ages and metallicities. At the limit, indices could be as narrow as a few tenths of an Ångström, but they will be accessible only to a few instruments. Also, the exposure time would have to be very high to obtain a high $S / N$ ratio. Moreover, stellar velocity dispersion broadens the lines $\left(50 \mathrm{~km} \mathrm{~s}^{-1}\right.$ corresponding to $F W H M=2.0 \AA$ at $5000 \AA$ for example). Extremely narrow indices would turn out to be unusable. For these reasons, we restrain our search to $2 \AA$-wide indices. In order to disentangle age and metallicity, we tried to build couples of indices sensitive to either age or metallicity, producing the most orthogonal and regular grids in the age- $Z$ plane, with the smallest measurement errors at a fixed mean $S / N$ ratio (hereafter 50). Indeed, as emphasized by Cardiel et al. (2003), this association of small measurement errors (which depend directly on the definitions of the indices) with an orthogonal grid is the most promising combination for disentangling age and metallicity signatures. The errors in index measurements are estimated following the formulae of Cardiel et al. (1998). 

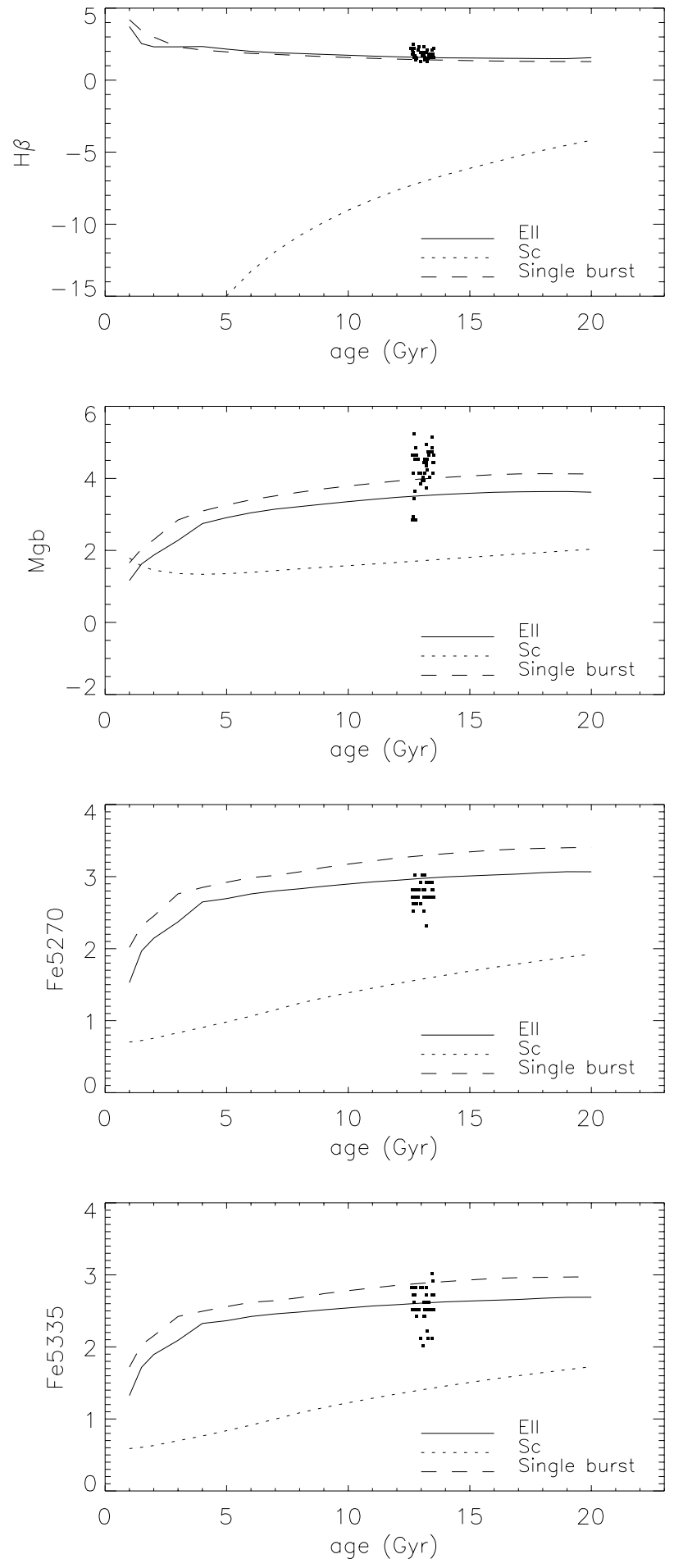

Fig. 14. Lick indices of PEGASE-HR models for elliptical, Sc and single burst galaxies together with data from local elliptical galaxies (Trager et al. 2000). The age of the data is arbitrarily set by us to $13 \pm 0.5 \mathrm{Gyr}$, randomly distributed (for the clarity of the plot). The presence of emission lines associated to ongoing star formation explains the low values of the $\mathrm{H} \beta$ index predicted for evolved spiral galaxies.

\subsection{Evolution of the pseudo-continuum}

The definition of the continuum is an important aspect of any index definition. The continuum can be defined, as in
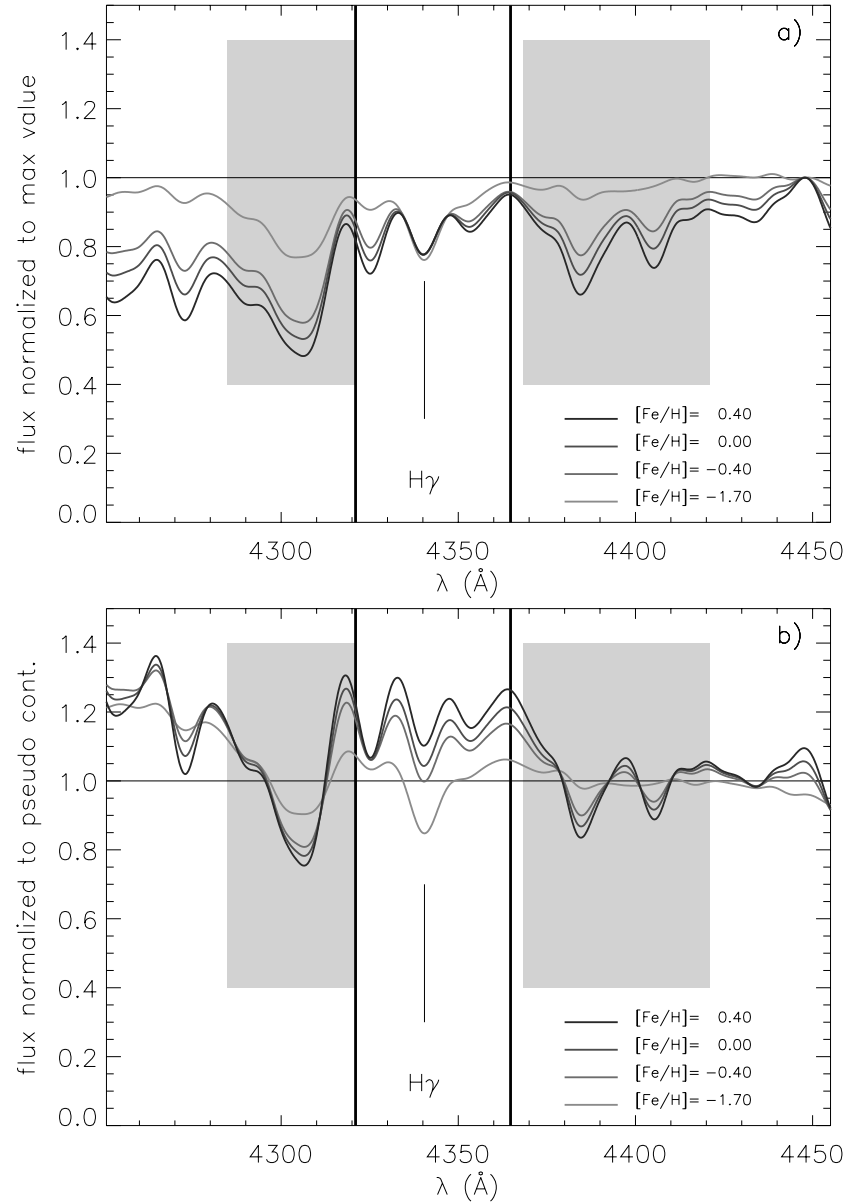

Fig. 15. Evolution of the $\mathrm{H} \gamma$ line as a function of metallicity for a 10 Gyr-old starburst: a) normalization at $4448 \AA$; b) normalization to the Lick $\mathrm{H} \gamma_{\mathrm{A}}$ pseudo-continuum. In both panels, the spectrum is degraded to the Lick resolution. Grey zones represent the $\mathrm{H} \gamma_{\mathrm{A}}$ Lick blue and red continuum definitions and solid vertical lines delimit the $\mathrm{H} \gamma_{\mathrm{A}}$ feature passband.

Bica \& Alloin (1986a), by the upper envelope of the spectrum, but is then strongly sensitive to noise and emission lines. The Lick indices define a "pseudo-continuum": a straight line between the mean fluxes in a blue and a red window. This choice is more robust and is convenient for the analysis of observations. The Lick normalization is empirical because the pseudocontinuum may evolve by itself with age and metallicity of the underlying populations. Figure 15 shows this effect on the $\mathrm{H} \gamma$ line. SSP spectra at various metallicities are presented at 10 Gyr with the Lick index resolution. Two normalizations are presented:

a) at $4448 \AA$ (top panel); the blue part of the Lick pseudocontinuum dramatically changes because it falls in the $G$ band;

b) at the Lick pseudo-continuum (bottom panel); the variations seem to reside in the $\mathrm{H} \gamma$ line itself.

Depending on normalization, conclusions may strongly differ. 
Table 2. Various $\mathrm{H} \gamma$ indices and resolutions (last column). $\mathrm{H} \gamma \_$VHR and $\mathrm{H} \gamma \_\mathrm{Z}$ are the new ones that we propose (see Sects. 5.3 and 5.4).

\begin{tabular}{|c|c|c|c|c|}
\hline Name & Feature & Blue band & Red band & $F W H M(\AA)$ \\
\hline $\mathrm{H} \gamma \_\mathrm{F}$ & 4332.5004353 .500 & 4284.7504321 .000 & 4356.0004386 .000 & 9.5 \\
\hline $\mathrm{H} \gamma \_\mathrm{HR}$ & 4338.6074342 .347 & 4333.0004335 .000 & 4348.0004350 .000 & 2.7 \\
\hline $\mathrm{H} \gamma \_\mathrm{Vaz}$ & 4332.0004352 .250 & 4331.0004340 .750 & 4359.2504368 .750 & 6.8 \\
\hline $\mathrm{H} \gamma_{-}$VHR & 4337.6004341 .200 & 4327.6004336 .400 & 4348.8004366 .800 & 2.0 \\
\hline $\mathrm{H} \gamma \_\mathrm{Z}$ & 4351.0004354 .000 & 4318.0004322 .500 & 4362.0004365 .000 & 2.0 \\
\hline
\end{tabular}

Table 3. Sensitivity of line equivalent widths to age (1-13 Gyr) and metallicity $([\mathrm{Fe} / \mathrm{H}]=-1.7$ to 0.4$) . \Delta_{[\mathrm{Fe} / \mathrm{H}]}$ represents the fractional change in $[\mathrm{Fe} / \mathrm{H}]$ needed to produce a $10 \%$ variation of the index value at the zero point $\left(Z_{\odot}, 12 \mathrm{Gyr}\right) . \Delta_{\text {age }}$ is the similar quantity computed with the age. $\frac{\Delta_{\text {age }}}{\Delta_{[\mathrm{Fe} / \mathrm{H}]}}$ is the ratio of fractional change in age to the fractional change in $[\mathrm{Fe} / \mathrm{H}]$ required to produce the same change in the index value (Worthey 1994; Jones \& Worthey 1995). High absolute values correspond to high sensitivity to $Z$. This quantity is evaluated around the $\left(Z_{\odot}, 12 \mathrm{Gyr}\right)$ zero point. The last column reproduces the values in Table 6 of Worthey (1994) and in Table 2 of Jones \& Worthey (1995).

\begin{tabular}{lcccc}
\hline \hline Index & $\Delta_{[\mathrm{Fe} / \mathrm{H}]}$ & $\Delta_{\text {age }}$ & $\frac{\Delta_{\mathrm{age}}}{\Delta_{[\mathrm{Fe} / \mathrm{H}]}}$ & $\left(\frac{\Delta_{\text {age }}}{\Delta_{[\mathrm{FF} / \mathrm{H}]}}\right)_{\mathrm{W}, \mathrm{J}}$ \\
\hline $\mathrm{Fe} 5335$ & 0.41 & 0.75 & 1.8 & 2.8 \\
$\mathrm{Fe} 5270$ & 0.42 & 0.74 & 1.8 & 2.3 \\
$\mathbf{H} \gamma_{-} \mathbf{Z}$ & 0.73 & 1.03 & 1.4 & \\
$\mathrm{Mg}$ & 0.55 & 0.56 & 1.0 & 1.7 \\
$\mathrm{H} \delta_{\mathrm{A}}$ & 0.16 & 0.14 & 0.9 & 1.1 \\
$\mathrm{H} \gamma_{\mathrm{A}}$ & 0.36 & 0.31 & 0.9 & 1.0 \\
$\mathrm{H} \gamma_{F}$ & 0.23 & 0.15 & 0.7 & \\
$\mathrm{H} \gamma_{-}$Vaz & -3.04 & -0.42 & 0.1 & \\
$\mathrm{H} \beta$ & -1.62 & -0.26 & 0.2 & 0.6 \\
$\mathrm{H} \gamma_{-} \mathrm{HR}$ & 6.50 & -1.06 & -0.2 & 0.0 \\
$\mathbf{H} \gamma_{-} \mathbf{V H R}$ & 11.05 & -1.06 & -0.1 & \\
\hline
\end{tabular}

\subsection{A systematic search method}

We have developed a method to find narrow indices, defined like the Lick indices, that could be good estimators of age or of metallicity. The first step is to compute SSP spectra for various metallicities, and various ages. Then, we normalize each spectrum to the maximum value within a sliding $50 \AA$-wide window. We thus obtain spectra with almost flat continua. Next, we measure, for each wavelength, the relative flux variations induced by either age changes or metallicity changes. We use the relative differences between these variations to select narrow feature windows and pseudo-continuum windows; we thereby define indices particularly sensitive to age or to metallicity. Since the $S / N$ ratio of the stellar library is finite, we take into account the uncertainties in the measurement of the flux variations relative to age or to metallicity. This leads us to select easily measurable indices only (i.e. with small measurement error bars).

\subsection{The age-sensitive $\mathrm{Hy}$ index $\mathrm{H} \gamma \_V H R$}

The $\mathrm{H} \gamma$ line, as a Balmer line, is a good age-sensitivity tracer. Moreover, unlike $\mathrm{H} \alpha$ and $\mathrm{H} \beta$, it is seldom filled in by nebular emission $\left(\mathrm{H} \gamma / \mathrm{H} \beta \simeq 0.5\right.$ in $\mathrm{H}_{\text {II }}$ regions). The upper panel of Table 2 presents various definitions for a $\mathrm{H} \gamma$ index (wavelengths and resolutions): $\mathrm{H} \gamma \_\mathrm{F}$ (Worthey, Lick resolution $\simeq 8 \AA$ ), $\mathrm{H} \gamma_{-}$Vaz (Vazdekis \& Arimoto 1999, $\sigma=200 \mathrm{~km} \mathrm{~s}^{-1}$ : $F W H M \simeq 6.8 \AA$ ), $\mathrm{H} \gamma \_$HR (Jones \& Worthey $1995, \sigma=$ $\left.80 \mathrm{~km} \mathrm{~s}^{-1}: F W H M \simeq 2.7 \AA\right)$. We hereafter propose the new index $\mathrm{H} \gamma_{-}$VHR (VHR standing for Very High Resolution) with $F W H M=2.0 \AA\left(\sigma \simeq 60 \mathrm{~km} \mathrm{~s}^{-1}\right)$, well suited to PEGASE-HR.

Figure 16 presents the comparison of the age- $Z$ grids built up with various $\mathrm{H} \gamma$ indices for the age axis, and with the Lick $\langle\mathrm{Fe}\rangle$ index for the $[\mathrm{Fe} / \mathrm{H}]$ axis. The last grid $\left(\mathrm{H} \gamma_{-} \mathrm{VHR}\right.$ vs. $\langle\mathrm{Fe}\rangle$ ) shows that the $\mathrm{H} \gamma_{-}$VHR index is particularly insensitive to the metallicity and allows a slightly better age estimation than $\mathrm{H} \gamma \_\mathrm{HR}$ at low metallicity. For old, solar-metallicity stellar populations, it is very similar to the $\mathrm{H} \gamma \_\mathrm{HR}$ index, but the error bar associated to its measurement (at $S / N=50$ on the plot) is smaller. It may be measured at high (>50) $S / N$ ratio with the new generation of instruments.

\subsection{A metal-sensitive $H \gamma$ index: $H \gamma \_Z$}

The high spectral resolution $(F W H M=2.0 \AA)$ makes it possible to present the new $\mathrm{H} \gamma \_\mathrm{Z}$ index, highly sensitive to metallicity. This might look surprising, but Vazdekis \& Arimoto (1999) and Jones \& Worthey (1995) already noted that many metallic lines are present in every Lick index. We call this new index $\mathrm{H} \gamma \_\mathrm{Z}$ because it is very close to the $\mathrm{H} \gamma$ line, but the central passband actually measures a chromium $(\mathrm{Cr})$ line. This index is voluntarily defined for a spectral resolution corresponding to $F W H M=2.0 \AA$. This narrow index is quite complementary to the index $\mathrm{H} \gamma_{-}$VHR: the passbands are very close to each other, and a single short spectrum is enough to perform an age- $Z$ diagnostic. The independence of the parameters traced by the two indices is exemplified in Fig. 17. The diagram $\mathrm{H} \gamma_{-}$VHR (agesensitive) vs. $\mathrm{H} \gamma \_\mathrm{Z}$ ( $Z$-sensitive) should be useful to interpret high-resolution spectra.

\section{5. $Z$ and age sensitivities}

We present in Table 3 updated values of the sensitivities to age and metallicity of some Lick indices $(\mathrm{H} \gamma, \mathrm{H} \beta, \mathrm{Fe} 5335$, Fe5270) and of the old and new $\mathrm{H} \gamma$ indices. We use the definition of Worthey (1994) for the quantity $\left(\Delta_{\text {age }} / \Delta_{[\mathrm{Fe} / \mathrm{H}]}\right)$ called 

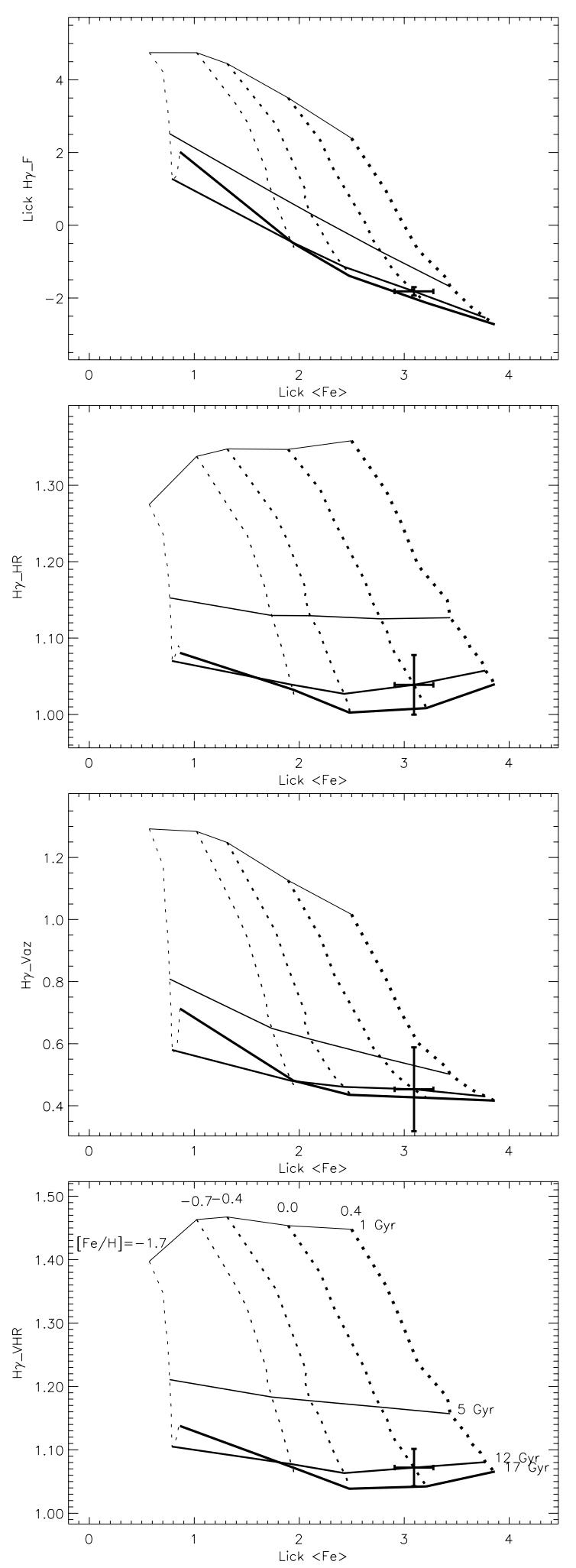

Fig. 16. $\mathrm{H} \gamma$ indices vs. $\langle\mathrm{Fe}\rangle$ Lick index. From top to bottom, the $\mathrm{H} \gamma$ indices are computed with the definitions of Worthey, Jones, Vazdekis, and our Very High Resolution index (see text for details). Error bars are computed from Cardiel et al. (1998) with an average $S / N$ ratio $=50$. Solid lines are iso-age sequences $(1,5,12,17 \mathrm{Gyr})$. Dotted lines are iso- $[\mathrm{Fe} / \mathrm{H}]$ sequences $(-1.7,-0.7,-0.4,0 ., 0.4)$. The thickness of the lines increases with age and $[\mathrm{Fe} / \mathrm{H}]$. Notice the particularly flat isoage sequences of the $\mathrm{H} \gamma_{-}$VHR index, which indicates a very low $Z$-sensitivity.

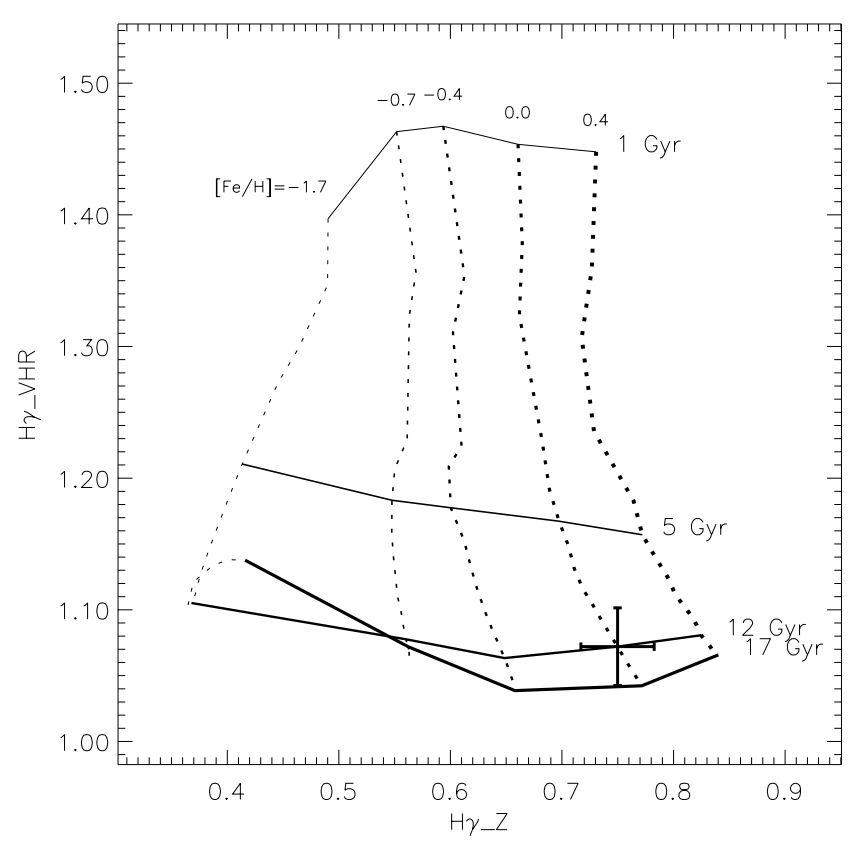

Fig. 17. $\mathrm{H} \gamma \_$VHR index (age tracer) vs. $\mathrm{H} \gamma \_\mathrm{Z}$ index (metallicity tracer) indices. Error bars are computed from Cardiel et al. (1998) with an average $S / N$ ratio of 50 . Same symbols as in Fig. 16.

" $Z$-sensitivity". It is the ratio of the fractional change in age to the fractional change in $Z$ needed to induce the same variation of the index value. A value close to zero means that the index is completely insensitive to metallicity variations, whereas a large absolute value indicates that the index is insensitive to age variations. We also give these fractional changes $\Delta_{\text {age }}$ and $\Delta_{[\mathrm{Fe} / \mathrm{H}]}$ for a $10 \%$ variation of the index value around the zero point $\left(Z_{\odot}, 12 \mathrm{Gyr}\right)$. A comparison to values obtained with previous models (Worthey 1994; Jones \& Worthey 1995) is also given in the last column. Our new index $\mathrm{H} \gamma_{-}$VHR is quite insensitive to metallicity variations around the zero point. The new index $\mathrm{H} \gamma \_\mathrm{Z}$ is very sensitive to variations of metallicity, even more than the $\mathrm{Mg}_{\mathrm{b}}$ index.

\section{Discussion and conclusion}

The code PEGASE-HR is a spectro-photometric evolution model of galaxies with very high spectral resolution in the optical. It inherits the PEGASE. 2 accurate modeling of galaxy evolution, as demonstrated by the compatibility of colors and low-resolution spectra between the two models. The ELODIE library is complete enough to give an extensive coverage of the HR diagram. Efforts are currently made to complete the library with low metallicity stars. Its quality depends on the estimation of stellar parameters with TGMET and on the interpolator which will be soon published. The ELODIE archive continues to grow from new observations, and the density of stars in the parameter space $\left(T_{\text {eff }}, \log _{10} g,[\mathrm{Fe} / \mathrm{H}]\right)$ increases. The improvement will be particularly noticeable for fast evolving stars and a better accuracy will be obtained for young starbursts (age < $10 \mathrm{Myr}$ ).

We checked that PEGASE-HR, degraded at low resolution, is in good agreement with other recent models: PEGASE.2, 
Bressan et al. (1996), Thomas et al. (2003), or Bruzual \& Charlot (2003). The predicted evolution of Lick indices for Balmer lines and metallic lines is compatible with most other models and observations. The small number of cool giant stars with super-solar metallicity in the ELODIE library and the uncertainty on their effective temperature makes the predictions of indices quite uncertain for old, metal-rich stellar populations. The influence of the non-solar enrichment for $\alpha$-elements in the ELODIE library needs more investigation.

We systematically explored the wavelength domain to find new high resolution indices, sensitive to either age or metallicity. Inside the passband of the classical $\mathrm{H} \gamma$ Lick index we find two very high resolution indices: one is sensitive to age only, and is quite similar to the index of Jones \& Worthey (1995). The other one is sensitive to metallicity only. These $2 \AA$-wide features are defined for velocity dispersions up to $60 \mathrm{~km} \mathrm{~s}^{-1}$. We note that these indices, despite a systematic investigation at high resolution, are not fundamentally different from the previously existing ones. This may indicate that future improvement will mainly come from high-resolution SED fitting rather than from classical indices.

This model is suited to the analysis of high resolution spectroscopic observations with rest-frame wavelengths falling in the 4000-6800 $\AA$ interval. In particular, the most recent spectrographs on large telescopes (GIRAFFE, VIMOS or ISAAC at the VLT, GMOS or GNIRS at the Gemini Observatory, EMACS on Magellan, TWIN at Calar Alto, ISIS at WHT...) will benefit from this model. We already presented tests of inversion methods showing their ability to separate disk and bulge components from a spectrum, deriving simultaneously their population characteristics and their kinematics (Prugniel et al. 2003; Ocvirk et al. 2003). This approach will be fundamental in many scientific applications, such as the study of bulges for which correcting the disc contamination is a prerequisite (Prugniel et al. 2001), or of high surface brightness dwarf objects.

The code, as well as SSPs, can be downloaded on the PEGASE web site at http://www.iap.fr/pegase/. Because the output spectra now contain ten times as many wavelength points as the PEGASE. 2 spectra, the SSPs are provided in FITS format. They contain all the information previously given by PEGASE. 2 outputs, except the colors. Measurements of Lick indices are included. A code to convert the FITS files into the PEGASE.2 ASCII format is also available for the user's convenience.

We stress that this new tool is unique for studying stellar populations in nearby and distant galaxies observed with the new high resolution spectrographs. Other improvements will come from multi-wavelength analyses, by combining PEGASE-HR optical spectra with far-ultraviolet and nearinfrared SEDs. Another improvement will come from the implementation of non-solar abundances in the evolution. For this purpose, we are presently measuring chemical abundances from the high resolution spectra of the ELODIE library.

Acknowledgements. We would like to thank the referee, Guy Worthey, for his very useful comments which helped improve the paper significantly. This work was supported in part by the French Programme National Galaxies.

\section{References}

Bica, E., \& Alloin, D. 1986a, A\&A, 162, 21

Bica, E., \& Alloin, D. 1986b, A\&AS, 66, 171

Bono, G., Caputo, F., Cassisi, S., Castellani, V., \& Marconi, M. 1997, ApJ, 489, 822

Bressan, A., Chiosi, C., \& Tantalo, R. 1996, A\&A, 311, 425

Brodie, J. P., \& Huchra, J. P. 1991, ApJ, 379, 157

Bruzual, G., \& Charlot, S. 2003, MNRAS, 344, 1000

Cardiel, N., Gorgas, J., Cenarro, J., \& Gonzalez, J. J. 1998, A\&AS, 127, 597

Cardiel, N., Gorgas, J., Sánchez-Blázquez, P., et al. 2003, A\&A, 409, 511

Cassisi, S., Castellani, M., \& Castellani, V. 1997, A\&A, 317, 108

Cayrel de Strobel, G., Soubiran, C., \& Ralite, N. 2001, A\&A, 373, 159

Cenarro, A. J., Cardiel, N., Gorgas, J., et al. 2001, MNRAS, 326, 959

Charbonnel, C., Meynet, G., Maeder, A., \& Schaerer, D. 1996, A\&AS, 115,339

Charlot, S., Worthey, G., \& Bressan, A. 1996, ApJ, 457, 625

Coluzzi, R. 1999, VizieR Online Data Catalog, 6071

Eisenstein, D. J., Hogg, D. W., Fukugita, M., et al. 2003, ApJ, 585, 694

Falcón-Barroso, J., Peletier, R. F., Vazdekis, A., \& Balcells, M. 2003, ApJ, 588, L17

Fioc, M. 1997, Ph.D. Thesis

Fioc, M., \& Rocca-Volmerange, B. 1997, A\&A, 326, 950

Fioc, M., \& Rocca-Volmerange, B. 1999

[arXiv: astro-ph/9912179]

Fuhrmann, K. 1998, A\&A, 338, 161

Fulbright, J. P. 2002, AJ, 123, 404

Golev, V., Prugniel, P., Simien, F., \& Longhetti, M. 1999, A\&AS, 136, 519

González, J. J. 1993, Ph.D. Thesis

Gorgas, J., Faber, S. M., Burstein, D., et al. 1993, ApJS, 86, 153

Green, E. M., Demarque, P., \& King, C. R. 1987, The revised Yale isochrones and luminosity functions (New Haven: Yale Observatory)

Houdashelt, M. L., Bell, R. A., Sweigart, A. V., \& Wing, R. F. 2000, AJ, 119, 1424

Huchra, J. P., Brodie, J. P., Caldwell, N., Christian, C., \& Schommer, R. 1996, ApJS, 102, 29

Jones, L. A. 1998, Ph.D. Thesis

Jones, L. A., \& Worthey, G. 1995, ApJ, 446, L31

Katz, D., Soubiran, C., Cayrel, R., Adda, M., \& Cautain, R. 1998, A\&A, 338, 151

Kuntschner, H., \& Davies, R. L. 1998, MNRAS, 295, L29

Kuntschner, H., Lucey, J. R., Smith, R. J., Hudson, M. J., \& Davies, R. L. 2001, MNRAS, 323, 615

Le Borgne, D., \& Rocca-Volmerange, B. 2002, A\&A, 386, 446

Leitherer, C., Alloin, D., Fritz-v. Alvensleben, U., et al. 1996, PASP, 108, 996

Leitherer, C., Schaerer, D., Goldader, J. D., et al. 1999, ApJS, 123, 3

Lejeune, T., Cuisinier, F., \& Buser, R. 1997, A\&AS, 125, 229

Lejeune, T., Cuisinier, F., \& Buser, R. 1998, A\&AS, 130, 65

Maraston, C. 1998, MNRAS, 300, 872

Maraston, C., Greggio, L., Renzini, A., et al. 2003, A\&A, 400, 823

Ocvirk, P., Lançon, A., Pichon, C., et al. 2003, in The Evolution of Galaxies. From Simple Approaches to Self-Consistent Models, ed. G. H. et al. (Kluwer), in press 
Prugniel, P., Maubon, G., \& Simien, F. 2001, A\&A, 366, 68

Prugniel, P., Simien, F., Fioc, M., et al. 2003, in Star Formation Through Time, ASP Conf. Ser., 297, 281

Prugniel, P., \& Soubiran, C. 2001a, A\&A, 369, 1048

Prugniel, P., \& Soubiran, C. 2001b, VizieR Online Data Catalog, 3218

Prugniel, P., \& Soubiran, C. 2004, in prep.

Ridgway, S. T., Joyce, R. R., White, N. M., \& Wing, R. F. 1980, ApJ, 235, 126

Rose, J. A., Bower, R. G., Caldwell, N., et al. 1994, AJ, 108, 2054

Salasnich, B., Girardi, L., Weiss, A., \& Chiosi, C. 2000, A\&A, 361, 1023

Salpeter, E. E. 1955, ApJ, 121, 161

Schaller, G., Schaerer, D., Meynet, G., \& Maeder, A. 1992, A\&AS, 96, 269

Sekiguchi, M., \& Fukugita, M. 2000, AJ, 120, 1072
Soubiran, C., Bienaymé, O., \& Siebert, A. 2003, A\&A, 398, 141

Soubiran, C., Katz, D., \& Cayrel, R. 1998, A\&AS, 133, 221

Thomas, D., Maraston, C., \& Bender, R. 2003, MNRAS, 339, 897

Trager, S. C., Faber, S. M., Worthey, G., \& González, J. J. 2000, AJ, 120, 165

Trager, S. C., Worthey, G., Faber, S. M., Burstein, D., \& Gonzalez, J. J. 1998, ApJS, 116, 1

Tripicco, M. J., \& Bell, R. A. 1995, AJ, 110, 3035

Vandenberg, D. A. 1985, ApJS, 58, 711

Vazdekis, A., \& Arimoto, N. 1999, ApJ, 525, 144

Worthey, G. 1994, ApJS, 95, 107

Worthey, G., Faber, S. M., Gonzalez, J. J., \& Burstein, D. 1994, ApJS, 94, 687

Worthey, G., \& Ottaviani, D. L. 1997, ApJS, 111, 377

Yi, S. K. 2003, ApJ, 582, 202 